PUPT-2148

\title{
Flux Vacua and Branes of the Minimal Superstring
}

\author{
Nathan Seiberg ${ }^{1}$ and David Shih ${ }^{2}$ \\ ${ }^{1}$ School of Natural Sciences, Institute for Advanced Study, Princeton, NJ 08540 USA \\ ${ }^{2}$ Department of Physics, Princeton University, Princeton, NJ 08544 USA
}

\begin{abstract}
We analyze exactly the simplest minimal superstring theory, using its dual matrix model. Its target space is one dimensional (the Liouville direction), and the background fields include a linear dilaton, a possible tachyon condensate, and RR flux. The theory has both charged and neutral branes, and these exhibit new and surprising phenomena. The smooth moduli space of charged branes has different weakly coupled boundaries in which the branes have different RR charges. This new duality between branes of different charges shows that the semiclassical notion of localized charge is not precise in the quantum theory, and that the charges of these branes can fluctuate. Correspondingly, the RR flux in some parts of target space can also fluctuate - only the net flux at infinity is fixed. We substantiate our physical picture with a detailed semiclassical analysis of the exact answers. Along the way, we uncover new subtleties in super-Liouville theory.
\end{abstract}

December, 2004 


\section{Introduction}

Minimal superstring theories, or $(p, q)$ superminimal CFT coupled to $\mathcal{N}=1$ superLiouville theory, are interesting toy models of string theory. Dual to certain large $N$ matrix models [1] [12], they have a precise and tractable nonperturbative definition. (For recent work on minimal superstrings, see [13]19].) Along with their bosonic cousins, they exhibit many important stringy phenomena, such as D-branes, holography and open/closed duality. In addition, the minimal superstring allows us to study interesting phenomena associated with RR flux and RR charge. The study of such flux vacua is interesting, among other reasons, because of their importance in the landscape of more generic string vacua.

In this paper, we will focus on the simplest minimal superstring theory, corresponding to $(p, q)=(2,4)$. We will use the exact matrix model description to study nonperturbative aspects of the theory in RR flux backgrounds. Specializing to $(p, q)=(2,4)$ simplifies the analysis considerably. In particular, it allows us to use interchangeably the $0 \mathrm{~A}$ and $\mathrm{OB}$ formulations of the theory, because in this case the two are equivalent [13]. We will see that some observables are easier to analyze in one formulation or the other.

One of the nice features of minimal string theory is that it allows us to study in great detail the interplay between various dual descriptions - worldsheet, target space, and matrix model - of the theory. These descriptions all have their strengths and weakness. For instance, calculations are easiest to carry out using the exact matrix model description, but their physical interpretation is often obscure. Conversely, the physics is clearest in the target space description, as it resembles that of more generic models, but calculations are often impossible using this description. Finally, the worldsheet formalism allows us to analyze the semiclassical physics to all orders in $\alpha^{\prime}$, but the higher order quantum corrections are not easy to calculate, and the exact answers are impossible to find.

Since our ultimate goal is to extract target space physics from the matrix model, let us describe in some detail the system from the target space perspective, following closely [13]. The target space consists of a single dimension $\phi$, described by the Liouville field on the worldsheet. The linear dilaton background for $\phi$ means that the string coupling depends on $\phi$ as

$$
g_{s}=e^{\frac{Q \phi}{2}}=e^{\frac{3 \phi}{2 \sqrt{2}}}
$$

where $Q=\frac{3}{\sqrt{2}}$ is the linear dilaton slope. Thus $g_{s}$ is zero at $\phi \rightarrow-\infty$ and grows exponentially as $\phi \rightarrow+\infty$. We will refer to these as the weak and strong coupling regions, respectively. 
The target space fields consist of the closed-string tachyon $T(\phi)$ and the RR scalar $C(\phi)$. In the vacuum we have

$$
T(\phi)=\mu e^{b \phi}=\mu e^{\frac{\phi}{\sqrt{2}}}
$$

where $\mu$ is referred to as the worldsheet bulk cosmological constant, and $b=\frac{1}{\sqrt{2}}$ is the worldsheet Liouville coupling constant. In order to examine the value of $C(\phi)$ in the vacuum, we consider the leading term in its Lagrangian [20,13]

$$
\mathcal{L}_{C}=\frac{1}{2 \sqrt{2}} e^{-2 T}\left(\partial_{\phi} C\right)^{2}
$$

This Lagrangian has the following symmetries: it is invariant under charge conjugation $C \rightarrow-C$ and under constant shifts of $C$. The latter gives rise to a conserved current

$$
q=e^{-2 T} \partial_{\phi} C
$$

We identify it as RR flux, which originates from RR charge $q$.

The solutions to the equations of motion of (1.3) are

$$
C(\phi)=C_{0}+q \int^{\phi} e^{2 T\left(\phi^{\prime}\right)} d \phi^{\prime}
$$

where $q$ is the background flux (1.4). The norm of the small fluctuations of $C$ is derived from (1.3), $\|\delta C\|^{2}=\int d \phi e^{-2 T}(\delta C)^{2}$. It determines the nature of the various possible fluctuations. For example, for positive $\mu$ the mode $C_{0}$ in (1.5) is (delta function) normalizable. This fluctuating mode allows us to explore the total charge of the system. This is to be contrasted with the flux term $q \int^{\phi} e^{2 T\left(\phi^{\prime}\right)} d \phi^{\prime}$ which is not normalizable as $\phi \rightarrow+\infty$. Such deformations were identified in 21] as arising from branes which are localized at infinity [22]. In the worldsheet description these are charged ZZ branes [23,24]. For negative $\mu$, things are slightly different. Here the flux term is normalizable in the strong coupling region, showing that the flux does not originate from a charged brane at infinity. The fact that this term is linearly divergent as $\phi \rightarrow-\infty$ shows that it is controlled by boundary conditions in the asymptotic weak coupling end, and in the worldsheet description of the theory it is described by a vertex operator (the RR ground state). By contrast, the solution $C_{0}$ is not normalizable at the strong coupling end, and therefore it does not correspond to an allowed vertex operator. It cannot fluctuate and it labels a superselection sector. However, because of the shift symmetry of $C$, the physical answers are independent of $C_{0}$. This is similar to the situation with spontaneous symmetry breaking. The novelty 
here, which happens because of the special coupling (1.3), is that we have spontaneous symmetry breaking in one dimension.

For positive $\mu$, the flux term in (1.5) arises from charged branes at infinity, so we cannot explore its contribution to the classical action without knowing more about these branes. We will do this below. However, for negative $\mu$, we can simply substitute the classical solution (1.5) into (1.3) to find 13

$$
S_{C}=\frac{1}{2 \sqrt{2}} q^{2} \int_{-\infty}^{\infty} d \phi \exp \left(2 \mu e^{\frac{\phi}{\sqrt{2}}}\right)=\frac{1}{2} q^{2} \log (\Lambda /|\mu|)
$$

where $\Lambda$ is a cutoff on $\phi$ in the weak coupling region. Later we will compare this with the matrix model results.

Besides analyzing bulk observables in flux backgrounds, we will also study D-branes and their interplay with the flux. Minimal superstring theory has both charged and neutral stable branes, which in the Liouville literature are called FZZT branes [25,26]. Their superLiouville version was explored in [23,24]. These branes are labelled by the value of the worldsheet boundary cosmological constant $\mu_{B}$. In target space, they have an open string tachyon whose value

$$
T_{\text {open }}(\phi)=\mu_{B} e^{\frac{b \phi}{2}}=\mu_{B} e^{\frac{\phi}{2 \sqrt{2}}}
$$

interpolates between $T_{\text {open }}=0$ as $\phi \rightarrow-\infty$ and $T_{\text {open }}= \pm \infty$ as $\phi \rightarrow+\infty$. The minisuperspace wavefunction $\Psi_{\text {open }}(\phi) \sim e^{-\left(T_{\text {open }}\right)^{2}}$ [20 means that we can think of these branes as semiextended, stretching from the weak coupling region to a point $\phi=\phi_{0} \sim-2 \sqrt{2} \log \left|\mu_{B}\right|$, where they dissolve away. We will argue below that semiclassically, the charged branes carry a half-unit of RR charge,

$$
q_{b}=\frac{1}{2} \operatorname{sign}\left(\mu_{B}\right)
$$

localized around $\phi=\phi_{0}$. Together with flux $q$ in the strong coupling region, this gives rise to flux

$$
q_{w e a k}=q+q_{b}
$$

in the weak coupling region. The wavefunction of the brane is shown together with its localized charge in figure 1. 


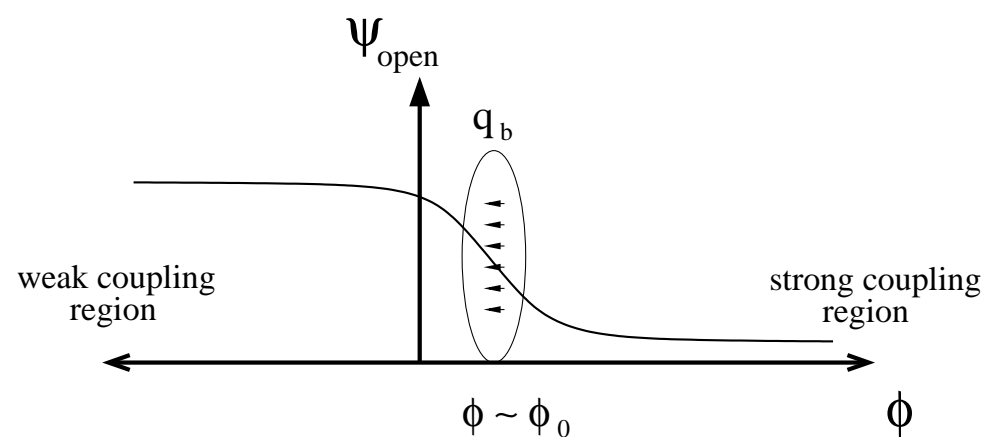

Fig. 1: The minisuperspace wavefunction for the charged FZZT brane, along with its localized charge. The brane comes in from the weak coupling region $\phi \rightarrow-\infty$ and dissolves at an intermediate point $\phi=\phi_{0} \sim-\log \left|\mu_{B}\right|$. By varying $\mu_{B}$, we can bring the tip of the brane into the weak or strong coupling region.

It may seem strange that we can change the charge of the brane by changing the sign of $\mu_{B}$. According to (1.9), this would also appear to change the value of $q_{\text {weak }}$. But for $\mu_{B} \approx 0$, the charge of the brane is localized in the strong-coupling region, and it is hard to see how varying $\mu_{B}$ by a small amount could affect the value of the flux in the weak-coupling region. This suggests that as $\mu_{B}$ is varied across zero, the value of the flux in the strong coupling region changes by one unit, in such a way that $q_{\text {weak }}=q+q_{b}=\left(q+2 q_{b}\right)-q_{b}$ is preserved. But this means that neither the flux in the strong coupling region nor the charge of the brane is well-defined! At large $\left|\mu_{B}\right|$, these quantities freeze to their semiclassical values, but in general they can fluctuate. Only $q_{\text {weak }}$, the flux in the weak-coupling region, is unambiguous.

Below we will discuss these phenomena in detail. We will also see how this qualitative target space picture is realized very quantitatively in the dual matrix model. In the matrix model description, we start with a closed-string background labelled by $q$, and there are initially two charged branes $B_{s}(x), s= \pm \frac{1}{2}$, which lead to flux

$$
q_{w e a k}=q+s
$$

in the weak-coupling region. The parameter $x$ which is natural in the matrix model is related to $\mu_{B}$ by

$$
x=i \mu_{B}
$$

So real $\mu_{B}$ corresponds to $x$ along the imaginary axis. Notice that the branes in the matrix model are essentially labelled by the flux in the weak coupling region (1.10), which is welldefined. This is to be expected, since the matrix model provides an exact description of 
the system. Now, using differential equations derived from the matrix model, it is possible to analyze the exact partition functions

$$
\psi_{ \pm}(x)=\frac{\left\langle B_{ \pm 1 / 2}(x)\right\rangle_{\mu, q}}{\mathcal{Z}(\mu, q)}
$$

of the charged branes. Here $\left\langle B_{ \pm 1 / 2}(x)\right\rangle_{\mu, q}$ is the unnormalized FZZT partition function (defined by the matrix integral), while $\mathcal{Z}(\mu, q)$ is the bulk partition function. We will show that these partition functions satisfy the nontrivial identity

$$
\left\langle B_{s}(x)\right\rangle_{\mu, q}=\left\langle B_{-s}(x)\right\rangle_{\mu, q+2 s}
$$

This identity substantiates the target space picture described above in a very precise way. It says that there is really only one brane for each $x$, and that we can think of this brane as either having charge $s$ in background flux $q$, or charge $-s$ in background flux $q+2 s$. The only unambiguous quantity is the net flux $q_{\text {weak }}=q+s$ at weak coupling.

These charged FZZT branes are analogous to the unstable branes of the critical string (for a review and a list of references see [27]). In addition to these branes our system also has neutral extended branes. These are analogous to the unstable brane-anti-brane states of the critical string. The open string tachyon on these neutral branes is complex and its phase is a gauge degree of freedom. Unlike their critical string counterparts, our charged and neutral branes are stable because the open string tachyon on them is actually massive.

The outline of the paper is as follows. In section 2 we will review and extend the analysis of the closed-string sector of our system. Semiclassically, it has two phases, distinguished by the sign of the cosmological constant $\mu$, and separated by a third-order phase transition [1]. The exact theory, however, does not exhibit a transition; rather, the interpolation from positive to negative $\mu$ is smooth [2-77,13]. This has an interesting consequence in backgrounds with nonzero flux $q$ : as $\mu$ is varied, there is a smooth transition from a phase where the flux comes from charged branes at infinity, to a phase where the flux comes from closed strings. Finally, we derive in section 2 various nontrivial identities for the bulk free energy that are necessary for the ensuing analysis of the D-branes.

In section 3 we review some of the facts of the worldsheet theory, focusing on the FZZT branes in super-Liouville theory. In particular, we will present some new terms which have to be added to the boundary states of some of the branes. These additions are motivated by our results in later sections. 
In sections 4 and 5, we study the charged and neutral D-branes of the system. Using differential equations derived from the matrix model, we will analyze their partition functions in various limits. As in [28], the exact answers are entire functions of $x$, but due to Stokes' phenomenon the semiclassical limit exhibits monodromy. We will see that in the superstring, Stokes' phenomenon is much more intricate and has additional physical consequences not present in the bosonic string. This additional structure arises largely from the possibility of having RR charge and flux in the minimal superstring. Thus, the study of the exact answers will lead to a much better understanding of background RR fluxes and their interplay with the various kinds of D-branes of the theory.

Finally, a few appendices are devoted to various technical details and relations to different points of view.

\section{The closed-string sector}

\subsection{The equations}

In this section, we will review some results on the closed-string sector of our model, following closely the presentation in 13 . 11 The observables of the closed-string sector depend on the values of the cosmological constant $\mu$ and the RR flux $q$. Using the duality with the matrix model (which we review in appendix A), one can derive differential equations - called the "string equations" - for the bulk partition function $\mathcal{Z}(\mu, q)$. These equations are most conveniently formulated in terms of a function $r=r(\mu, q)$, which is related to the free energy $F(\mu, q)=-\log \mathcal{Z}(\mu, q)$ via

$$
\frac{1}{2} r^{2}=F^{\prime \prime}
$$

In terms of $r$, the string equations take the form:

$$
\begin{aligned}
& r^{\prime \prime}-\mu r-r^{3}+r \beta^{\prime 2}=0 \\
& r^{2} \beta^{\prime}=q
\end{aligned}
$$

1 As mentioned in the introduction, the 0A and 0B GSO projections are equivalent for $(p, q)=$ $(2,4)$, so we will not distinguish between them. In [13], $0 \mathrm{~A}$ and $\mathrm{OB}$ were identified with a change in sign of the cosmological constant $\mu$. We will stick to the 0A sign conventions of [13]. 
where' is shorthand for $\partial_{\mu}$ and $\beta=\beta(\mu, q)$ is an auxiliary function. As we will see in a moment, $\beta$ is useful because it makes manifest certain symmetries of the system. Of course, it can be easily eliminated, leading to a differential equation for $r$ alone:

$$
r^{\prime \prime}-\mu r-r^{3}+\frac{q^{2}}{r^{3}}=0
$$

We recognize this as a modified version of the Painlevé II equation. Note that the equations (2.2) can be interpreted as the equations of motion derived from the "Lagrangian"

$$
\frac{1}{2}\left(r^{\prime}\right)^{2}-\frac{1}{2} r^{2} \beta^{\prime 2}+\frac{1}{2} \mu r^{2}+\frac{1}{4} r^{4}+q \beta^{\prime}
$$

where we view $\beta^{\prime}$ as an independent variable. Integrating out $\beta^{\prime}$ results in a Lagrangian that describes a particle with "time" $\mu$ and "position" $r(\mu)$, moving in a one-dimensional potential

$$
V(r)=-\left(\frac{1}{4} r^{4}+\frac{1}{2} \mu r^{2}+\frac{q^{2}}{2 r^{2}}\right)
$$

From the form of the potential, it is not hard to see that for every $q$ there exists a solution to the equations of motion which is everywhere real and positive, and which interpolates smoothly between $r=+\infty$ at $\mu=-\infty$ and $r=0$ at $\mu=+\infty$. These are the physical solutions that we will focus on henceforth.

The equations of motion (2.2) and the action (2.4) are invariant under the charge conjugation symmetry: $\beta \rightarrow-\beta, q \rightarrow-q$. It follows that

$$
r(\mu, q)=r(\mu,-q)
$$

(Actually, the symmetry could have related two different solutions, but this is not the case for the solution satisfying our boundary conditions.) In addition, (2.2) and (2.4) are also invariant under shifts of $\beta$ by a constant. The parameter $q$, which appears in (2.4) as a "topological term," can be interpreted as the conserved charge associated with this symmetry. Below we will limit ourselves to $q \in \mathbb{Z}$ and will make a few comments about more general values.

Now let us explore some of the properties of these equations. For this, it is convenient to define

$$
Z_{ \pm}=r e^{\mp \beta}=r e^{\mp \int \frac{q}{r^{2}} d \mu}
$$

Notice that the charge conjugation symmetry (2.6) implies

$$
Z_{+}(\mu, q)=Z_{-}(\mu,-q)
$$


The solutions to the string equation satisfy a nontrivial identity involving $Z_{ \pm}$[6,29]

$$
r(\mu, q \pm 1)^{2}=r(\mu, q)^{2}-2\left(\log Z_{ \pm}(\mu, q)\right)^{\prime \prime}
$$

It is amusing to view (2.9) as a discrete version of $\mathrm{KdV}$ flow, with the discrete parameter $q$ playing the role of the coupling constant. To prove (2.9), simply check that the square root of the RHS solves the string equation (2.3) with $q \rightarrow q \pm 1$. Of course, this does not guarantee that the solution $r(\mu, q \pm 1)$ generated in this way is physical, in the sense described below (2.5). In the next subsection, we will see that $q \in \mathbb{Z}$ is the only consistent set of $q$ for which this is always true.

Using (2.1), we can integrate (2.9) twice and exponentiate to obtain?

$$
Z_{ \pm}(\mu, q)=e^{F(\mu, q)-F(\mu, q \pm 1)}=\frac{\mathcal{Z}(\mu, q \pm 1)}{\mathcal{Z}(\mu, q)}
$$

Thus $Z_{ \pm}(\mu, q)$ can be thought of as the expectation value of an operator which changes $q \rightarrow q \pm 1$. Finally, let us mention a trivial consequence (2.10):

$$
Z_{+}(\mu, q-1) Z_{-}(\mu, q)=1
$$

This expression will be useful below.

\subsection{Semiclassical limit}

Since $g_{s} \sim|\mu|^{-3 / 2}$ according to (1.1) and (1.2), the semiclassical $g_{s} \rightarrow 0$ limit corresponds to the $|\mu| \rightarrow \infty$ limit. For $q=0$ the leading terms in the semiclassical expansion are

$$
\begin{aligned}
& r(\mu, q=0)= \begin{cases}\sqrt{-\mu}\left(1+\mathcal{O}\left(\mu^{-3}\right)\right) & \mu<0 \\
\sqrt{2} A i(\mu)\left(1+\mathcal{O}\left(e^{-\frac{4 \mu^{3 / 2}}{3}}\right)\right) & \mu>0\end{cases} \\
& \beta(\mu, q=0)=0 \\
& Z_{ \pm}(\mu, q=0)=r(\mu, q=0)
\end{aligned}
$$

We have set $\beta=0$ using the freedom to shift $\beta$ by a constant, in order to preserve the charge conjugation symmetry (2.8) at $q=0$.

2 There are possible $q$-dependent integration constants here. We show in appendix B that these can be consistently set to zero. 
For nonzero $q$ the situation is more interesting. For negative $\mu$ (the two cut phase of the $0 \mathrm{~B}$ theory) the solution of the string equations (2.2) is

$$
\begin{aligned}
& r(\mu, q)=\sqrt{-\mu}\left(1+\frac{1-4 q^{2}}{8 \mu^{3}}+\mathcal{O}\left(\mu^{-6}\right)\right) \\
& \beta(\mu, q)=-q \log (-\mu)+\mathcal{O}\left(\mu^{-3}\right) \\
& Z_{ \pm}(\mu, q)=r e^{\mp \beta}=(-\mu)^{ \pm q+1 / 2}\left(1+\mathcal{O}\left(\mu^{-3}\right)\right)
\end{aligned}
$$

Here we have again used the freedom to shift $\beta$ by a constant in order to satisfy (2.11).

For positive $\mu$ (the one cut phase of the $0 \mathrm{~B}$ theory) the limit $q \rightarrow 0$ is not smooth. We will find it convenient throughout to parametrize this discontinuity in terms of a function

$$
\epsilon(q)= \begin{cases}-1 & q<0 \\ 0 & q=0 \\ +1 & q>0\end{cases}
$$

Then for nonzero $q$ we find

$$
\begin{aligned}
& r(\mu, q)=\sqrt{\frac{|q|}{\sqrt{\mu}}}\left(1-\frac{|q|}{4 \mu^{3 / 2}}+\mathcal{O}\left(\mu^{-3}\right)\right) \\
& \beta(\mu, q)=\epsilon(q) \frac{2 \mu^{3 / 2}}{3}+\frac{q}{2} \log (\mu)+\log B(q)+\mathcal{O}\left(\mu^{-3 / 2}\right) \\
& Z_{ \pm}(\mu, q)=r e^{\mp \beta}=B(q)^{\mp 1} \sqrt{|q|}(\mu)^{-\frac{1}{4} \mp \frac{q}{2}} e^{\mp \epsilon(q) \frac{2 \mu^{3 / 2}}{3}}\left(1+\mathcal{O}\left(\mu^{-3 / 2}\right)\right)
\end{aligned}
$$

Note that in order to find the leading order contributions to $Z_{ \pm}$we need to expand $\beta(\mu, q)$ (and hence $r(\mu, q)$ ) to the next to leading order. In (2.15) we have included a possible integration constant $B(q)$ in $\beta$. This cannot be set to zero, since we have already used this freedom at the $\mu \rightarrow-\infty$ end $(2.13)$. Instead, we can use the fact that $Z_{+}(\mu, q-1) Z_{-}(\mu, q)$ is independent of $\mu$ to determine $B(q)$ - since we have set $Z_{+}(\mu, q-1) Z_{-}(\mu, q)=1$ at $\mu \rightarrow-\infty$, the same must be true at $\mu \rightarrow+\infty$.

Using these asymptotic expansions we can, as promised, examine the compatibility between the identity (2.9) and the physical requirements on $r(\mu, q)$ described below (2.5). Consider what happens for $q \in(-1,0)$. If (2.9) holds, then for large positive $\mu$, using (2.15) gives $r(\mu, q+1)^{2} \sim-\frac{q+1}{\mu^{1 / 2}}<0$. But this violates the physical requirement on $r$ that it be everywhere real and positive. So if we want to include noninteger values of $q$, we are forced to discard the identity (2.9). Conversely, if we take

$$
q \in \mathbb{Z}
$$


then (2.9) is always satisfied. We will see below that (2.9) has desirable, physical consequences. Therefore, we will assume $q \in \mathbb{Z}$ throughout the paper.

It is also interesting to calculate from (2.13) and (2.15) the leading order terms in the perturbative expansion of the bulk free energy. Here we find 3

$$
F(\mu, q)= \begin{cases}-\frac{\mu^{3}}{12}+\frac{1}{8} \log (-\mu)-\frac{1}{2} q^{2} \log (-\mu)+\mathcal{O}\left(\mu^{-3}\right) & \mu<0 \\ \frac{2}{3}|q| \mu^{3 / 2}+\frac{1}{4} q^{2} \log \mu+A(q)+\mathcal{O}\left(\mu^{-3 / 2}\right) & \mu>0\end{cases}
$$

As a consistency check, notice that the leading-order $q$-dependent term for $\mu<0$ agrees precisely with the calculation (1.6) based on the target space action. From (2.17), we can learn about the physical origin of the flux $q$ in the two phases. In general, we expect the genus expansion

$$
F=\sum_{h, b} F_{h, b}|\mu|^{3(1-h)}\left(\frac{|q|}{|\mu|^{\frac{3}{2}}}\right)^{b}
$$

with $F_{h, b}$ independent of $\mu$ and $q$. Here $h$ is the number of handles in the worldsheet and $b$ is the number of boundaries. According to (2.17), the leading contribution for $\mu>0$ is from $h=0$ and $b=1$, i.e. the disk. The factor of $|q|$ indicates that there are $q$ charged branes on which the boundary of the worldsheet can end. The higher order corrections are also consistent with this picture. We identify these branes with the charged ZZ branes localized at strong coupling that exist in this phase. Meanwhile, for $\mu<0$ all the terms in the perturbative expansion (2.18) have even $b$. We interpret this to mean that they come from surfaces with no boundaries but with $2 b$ insertions of an RR vertex operator with coefficient $q$. For instance, the leading order $q^{2}$ term comes from two insertions of the RR vertex operator on the sphere.

Note that for $\mu$ positive, $q$ arises from charged instantons. However, it does not fluctuate, because it affects the flux at infinity. This is to be contrasted with the bosonic string, where the analogous contributions must be summed, because there the instantons do not carry charge. From (2.17), we also see that the instantons have action $\frac{2}{3} \mu^{3 / 2}$ in this phase. For $q=0$, the leading order nonperturbative effects (see $(2.12))$ are $\mathcal{O}\left(e^{-\frac{4 \mu^{3 / 2}}{3}}\right)$; thus, these are instanton-anti-instanton effects. As in the bosonic string, these instantonanti-instanton effects should be summed over because they do not have charge.

3 A similar discussion of the integration constants applies here. Since $F(\mu, q)$ is smooth for real $\mu$, we fix the integration constants (to zero) at $\mu \rightarrow-\infty$ using (2.10) and then the integration constant $A(q)$ is determined also for $\mu \rightarrow+\infty$. 
As we cross from positive to negative $\mu$, there is a transition in the strong-coupling region between the flux $q$ being generated by charged branes or simply being there without branes as sources. Since the exact answer is a smooth function of $\mu$, this transition must be smooth as well.

In the rest of the paper, we will study the extended branes of the minimal superstring known as FZZT branes on the worldsheet - and their interaction with RR flux backgrounds described in this section. We will see that these branes provide us with further interesting examples of smooth, nonperturbative transitions between seemingly different flux vacua.

\section{Worldsheet Description of FZZT Branes}

Before going on to describe the exact matrix model analysis of the FZZT branes, let us first review how they are described on the worldsheet. The worldsheet description will provide nontrivial checks of the exact answer, as well as shed light on its physical interpretation.

In the worldsheet description, D-branes are conveniently described using boundary states. The boundary states for the FZZT branes were first written down for superLiouville theory in [23,24], and they were later adapted to the minimal superstring in [20,13, 15]. They depend on the sign of $\mu$, and they are labelled by a parameter $\eta= \pm 1$ which determines the boundary condition on the worldsheet supercharge, $Q=i \eta \bar{Q}$. For $\mu>0$, they aret

$$
\begin{aligned}
& \left.\left.|\sigma, \eta=-1\rangle_{ \pm}=\int_{0}^{\infty} d P\left(\frac{\mu}{2}\right)^{i P}(\cos (2 P \sigma)|P, \eta=-1\rangle\rangle_{N S} \pm \cos (2 P \sigma)|P, \eta=-1\rangle\right\rangle_{R}\right) \\
& \left.|\sigma, \eta=+1\rangle=\sqrt{2} \int_{0}^{\infty} d P\left(\frac{\mu}{2}\right)^{i P} \cos (2 P \sigma)|P, \eta=+1\rangle\right\rangle_{N S}
\end{aligned}
$$

Here $|P, \eta\rangle\rangle_{N S, R}$ are the NS and R Ishibashi states, apart from an overall $P$ dependent normalization that we will ignore. The RR Ishibashi states in the branes with $\eta=-1$ show that they are charged, while branes with $\eta=+1$ are neutral.

Notice that the relative normalization between the $\eta=+1$ and $\eta=-1$ boundary states differs by a factor of $\sqrt{2}$. This factor of $\sqrt{2}$ originates from the ratio of the disk of the spin field Cardy state and the identity and fermion Cardy states in the Ising model.

4 Our conventions here differ slightly with those of [15]: $\sigma_{\text {here }}=\frac{\pi b}{2} \sigma_{\text {there }}, P_{\text {here }}=\frac{1}{b} P_{\text {there }}$, and $\mu_{\text {here }}=-2 \mu_{\text {there }}$. 
It is also common in the similar setup of non-BPS branes in the critical string [30,27]. We will confirm it below in the exact analysis.

The boundary states are most natural in terms of $\sigma$, but the physical parameter of interest is the boundary cosmological constant $\mu_{B}$. The relation between the two [23,24] depends on $\eta$ (and, as we will see, the sign of $\mu$ ):

$$
\mu_{B}=-i x= \begin{cases}\sqrt{\frac{\mu}{2}} \cosh \sigma & \eta=-1 \\ \sqrt{\frac{\mu}{2}} \sinh \sigma & \eta=+1\end{cases}
$$

In [15], this relation was interpreted as the boundary analogue of the (super)Bäcklund transformation, with $\sigma$ identified as the Dirichlet boundary condition on the Bäcklund field. Note that here we have also introduced $x=i \mu_{B}$, the parameter most natural in the matrix model description.

Using the boundary states, we can compute the genus zero one-point functions of physical vertex operators simply by substituting the appropriate value of $P$ (for details, see [15]). In particular, the one-point function of the cosmological constant operator (given by $P=-\frac{i}{2}$ ) is simply

$$
\left\langle\int d^{2} z e^{\frac{\phi(z)}{\sqrt{2}}}\right\rangle=\sqrt{\frac{\mu}{2}} \cosh (\sigma)
$$

for both signs of $\eta$. Integrating once with respect to $\mu$ holding $x$ fixed yields the FZZT disk amplitude [13,15]:

$$
D(x)= \begin{cases}-i\left(\frac{4}{3} x^{3}+\mu x\right) & \eta=-1 \\ -\frac{4}{3} \sqrt{2}\left(\frac{\mu}{2}-x^{2}\right)^{3 / 2} & \eta=+1\end{cases}
$$

Notice that, strictly speaking, the integral with respect to $\mu$ does not determine the $x^{3}$ term for $\eta=-1$. We will see how this is fixed below, when we study the disk for $\mu<0$.

The disk amplitudes can be written in the form $D(x)=\int^{x} y\left(x^{\prime}\right) d x^{\prime}$, with

$$
y(x)^{2}= \begin{cases}-\left(4 x^{2}+\mu\right)^{2} & \eta=-1 \\ -32 x^{2}\left(x^{2}-\frac{\mu}{2}\right) & \eta=+1\end{cases}
$$

The algebraic curve $y^{2}=y(x)^{2}$ defines a Riemann surface associated with each FZZT brane. This Riemann surface is a double cover of the complex $x$ plane. For $\eta=-1$, this surface breaks into two subsurfaces where $y= \pm y(x)$. The subsurfaces are connected by two singularities at imaginary $x= \pm \frac{\sqrt{-\mu}}{2}$. Meanwhile, for $\eta=+1$ the surface is irreducible, has branch points at $x= \pm \sqrt{\frac{\mu}{2}}$ (it is convenient to take the cuts to lie along the real $x$ axis), and has a singularity at $x=0$. It was shown in [15] how these surfaces provide 
a simple geometric interpretation for minimal superstring theory. In particular, the two subsurfaces with $\eta=-1$ represent the branes with the two different charges [15]. Below we will see how this classical picture is modified in the exact theory.

Now let us turn to the FZZT boundary states for $\mu<0$. These are given by

$$
\begin{aligned}
& |\sigma, \eta=-1\rangle_{ \pm}= \\
& \left.\left.\quad \int_{0}^{\infty} d P\left(-\frac{\mu}{2}\right)^{i P}(\cos (2 P \sigma)|P, \eta=-1\rangle\rangle_{N S} \mp i \sin (2 P \sigma)|P, \eta=-1\rangle\right\rangle_{R}\right) \pm \frac{1}{2} V_{R}|0\rangle \\
& \left.|\sigma, \eta=+1\rangle=\sqrt{2} \int_{0}^{\infty} d P\left(-\frac{\mu}{2}\right)^{i P} \cos (2 P \sigma)|P, \eta=+1\rangle\right\rangle_{N S}
\end{aligned}
$$

where now

$$
\mu_{B}=-i x= \begin{cases}\sqrt{-\frac{\mu}{2}} \sinh \sigma & \eta=-1 \\ \sqrt{-\frac{\mu}{2}} \cosh \sigma & \eta=+1\end{cases}
$$

Here $V_{R}$ is the RR vertex operator which creates one unit of flux. Its $\phi$ dependence in the $(-1 / 2,-3 / 2)$ picture is $\phi e^{Q \phi / 2}$, and in the $(-1 / 2,-1 / 2)$ picture it is simply $e^{Q \phi / 2}$. The extra term $\pm \frac{1}{2} V_{R}|0\rangle$ was missed in the literature. The exact analysis in the next section will show that it is necessary. Note that if not for this extra term, the state $|\sigma, \eta=-1\rangle_{+}$ would be identical to the state $|-\sigma, \eta=-1\rangle_{-}$. This was the basis of the claim in the literature that these two $\eta=-1$ FZZT branes are identical in this phase. The new term shows that this claim is in fact false. Instead, the worldsheet theory has two oppositely charged $\eta=-1$ branes, for both signs of $\mu$.

As before, we can derive the disk amplitude starting from the one-point function of the cosmological constant operators. This gives

$$
D(x)= \begin{cases}-\frac{4}{3} i\left(x^{2}+\frac{\mu}{2}\right)^{3 / 2} & \eta=-1 \\ -i \sqrt{2}\left(\frac{4}{3} x^{3}-\mu x\right) & \eta=+1\end{cases}
$$

Here we have determined the $x^{3}$ term and the overall normalization of the $\eta=+1$ disk amplitude as follows. When $|x|$ is large, the brane is far in the weak coupling region, so the disk amplitude should be insensitive to the sign of $\mu$, as this only affects the physics in the strong-coupling region. In the $\mu>0$ phase (3.4), the $\eta=+1$ disk becomes $D(x) \rightarrow$ $-i \sqrt{2}\left(\frac{4}{3} x^{3}-\mu x\right)$ at large $|x|$. This fixes the $x^{3}$ term and the overall normalization of the $\eta=+1$ disk in the $\mu<0$ phase, (3.8). A similar argument also fixes the $x^{3}$ term and the overall normalization of the $\eta=-1$ disk amplitude in (3.4), as promised. 
We can again write the disk amplitude as $D(x)=\int^{x} y\left(x^{\prime}\right) d x^{\prime}$ with

$$
y(x)^{2}= \begin{cases}-16 x^{2}\left(x^{2}+\frac{\mu}{2}\right) & \eta=-1 \\ -2\left(4 x^{2}-\mu\right)^{2} & \eta=+1\end{cases}
$$

Again, $y^{2}=y(x)^{2}$ defines Riemann surfaces for each $\eta$. Comparing with (3.5), it is obvious that the $\mu<0, \eta= \pm 1$ surfaces are essentially the same as the $\mu>0, \eta=\mp 1$ surfaces. However, we will see later that the different $\eta$ surfaces are distinguished by their response to nonzero $q$.

Let us also point out that the new term $\pm \frac{1}{2} V_{R}|0\rangle$ in (3.6) does not affect the disk amplitude. Therefore it does not affect the curve $y(x)$. However, since it distinguishes between $|\sigma, \eta=-1\rangle_{+}$and $|-\sigma, \eta=-1\rangle_{-}$, these two branes are not related by analytic continuation in $\sigma$, and they take values in two different but isomorphic surfaces. Below, we will return to this point.

In the next section, we will study the exact FZZT partition functions, which reduce to $e^{D(x)+\ldots}$ in the semiclassical limit. Since $D(x)$ takes values on a Riemann surface, the semiclassical limit exhibits monodromy. However, we will see that the exact answers are entire in the complex $x$ plane. As in [28], Stokes' phenomenon replaces the semiclassical Riemann surface with a single copy of the $x$ plane.

\section{The Charged Branes}

\subsection{The differential equations}

In this section, our goal is to study the exact partition functions

$$
\psi_{ \pm}=\psi_{ \pm}(x, \mu, q)=\frac{\left\langle B_{ \pm 1 / 2}(x)\right\rangle_{\mu, q}}{\mathcal{Z}(\mu, q)}
$$

for the charged branes $B_{ \pm 1 / 2}$ of our system. These are related to the $\eta=-1$ FZZT branes in the worldsheet description reviewed above. We will see, however, that the relationship is subtle in many ways.

The charged branes are most natural in the 0B language. Through the identification of the FZZT branes with the determinant operator of the 0B matrix model (see appendix A.1), one can derive the following differential equations for $\psi_{ \pm}$:

$$
\begin{aligned}
& \left(\partial_{\mu}-A_{\mu}\right) \Psi=\left(\partial_{\mu}-\left(\begin{array}{cc}
-i x & \frac{Z_{+}}{\sqrt{2}} \\
\frac{Z_{-}}{\sqrt{2}} & i x
\end{array}\right)\right) \Psi=0 \\
& \left(\partial_{x}-A_{x}\right) \Psi=\left(\partial_{x}-\left(\begin{array}{cc}
-i\left(4 x^{2}+r^{2}+\mu\right) & 2 \sqrt{2} x Z_{+}+\sqrt{2} i Z_{+}^{\prime} \\
2 \sqrt{2} x Z_{-}-\sqrt{2} i Z_{-}^{\prime} & i\left(4 x^{2}+r^{2}+\mu\right)
\end{array}\right)\right) \Psi=0
\end{aligned}
$$


where $\Psi=\left(\begin{array}{c}\psi_{+} \\ \psi_{-}\end{array}\right)$. These equations were studied extensively for $q=0$ in [31, and the generalization to nonzero $q$ is straightforward. The details are left to appendix A.1. Here let us just mention three consistency checks. First, notice that in the normalization where $Z_{ \pm}$have charges \pm 1 , the functions $\psi_{ \pm}$have charges $\pm 1 / 2$. This is consistent with the target space interpretation of the branes, and it justifies our notation $B_{ \pm 1 / 2}$ above. Second, it is important that the string equation (2.3) implies the flatness condition

$$
F_{x \mu}=\partial_{x} A_{\mu}-\partial_{\mu} A_{x}+\left[A_{\mu}, A_{x}\right]=0
$$

This in turn guarantees the compatibility of the two equations in (4.2). Third, the fact that $A_{\mu}$ and $A_{x}$ are regular for all (finite) $x \in \mathbb{C}$ and $\mu \in \mathbb{R}$ means that $\Psi(x, \mu, q)$ is an entire function of $x$ and is smooth for $\mu$ real. This agrees with the expectation that the branes should be single-valued with respect to the coupling constants.

In order to solve the differential equations (4.2), we must specify boundary conditions for $\Psi$. The two linearly independent solutions to (4.2) are distinguished by their behavior at large $|x|$, with one exponentially increasing and the other exponentially decreasing as $x \rightarrow+i \infty$. On the other hand, the physical solution must satisfy

$$
\lim _{x \rightarrow+i \infty} \Psi(x, \mu, q)=\lim _{x \rightarrow-i \infty} \Psi(x, \mu, q)=0
$$

This follows from the fact that the matrix model potential goes to $+\infty$ at $x \rightarrow \pm i \infty$ (see the discussion in appendix A.1). For $q=0$, the existence of a regular solution to (4.2) satisfying (4.4) was proven in [32]. We can extend the existence proof to all $q \in \mathbb{Z}$ by using the following nontrivial identity

$$
\psi_{-}(x, \mu, q)=Z_{-}(\mu, q) \psi_{+}(x, \mu, q-1)
$$

which relates solutions with different $q$. Note that we can also write $(4.5)$ as $\psi_{+}(x ; \mu, q)=$ $Z_{+}(\mu, q) \psi_{-}(x ; \mu, q+1)$ using (2.11). Starting from the physical solution at $q=0$, these identities generate solutions at $q= \pm 1$ with the correct asymptotics. This proves the existence of the physical solution for $q= \pm 1$, and continuing in this way we prove existence for all $q \in \mathbb{Z}$. In the process, we also determine the overall $q$-dependent normalization of $\psi_{ \pm}$which is left unfixed by (4.2).

The identity (4.5) has been studied before in the mathematical literature (see e.g. [33, 34 ) and is sometimes referred to as a Schlesinger or Bäcklund transformation. Below 
we will understand the physical interpretation of (4.5) in terms of D-branes and fluxes. Since the proof of (4.5) is rather technical, we reserve it for appendix B.

Another property of the solutions follows from the charge conjugation symmetry of the theory. As mentioned above, the bulk theory is invariant under $q \rightarrow-q$. Since $Z_{+}(\mu, q)=Z_{-}(\mu,-q)$, charge conjugation is also a symmetry of the differential equations (4.2), provided we interchange $\psi_{+}$with $\psi_{-}$and send $x \rightarrow-x$. However, a symmetry of the equations is not necessarily a symmetry of the solutions. Instead, charge conjugation could map one solution into another. Fortunately, this does not happen here. Charge conjugation must map the physical solution at $q$ into the physical solution at $-q$, since the unphysical solution is exponentially increasing at large imaginary $x$. Thus we conclude that

$$
\psi_{+}(-x, \mu, q)=\psi_{-}(x, \mu,-q)
$$

Let us explore some of the consequences of these identities. First, notice that we can use (4.5) to rewrite the differential equations as equations for $\psi_{+}$alone:

$$
\begin{aligned}
& \partial_{\mu} \tilde{\Psi}=\left(\begin{array}{cc}
-i x & \frac{1}{\sqrt{2}} r^{2} \\
\frac{1}{\sqrt{2}} & i x-\partial_{\mu} \log Z_{-}
\end{array}\right) \tilde{\Psi} \\
& \partial_{x} \tilde{\Psi}=\left(\begin{array}{cc}
-i\left(4 x^{2}+r^{2}+\mu\right) & r^{2}\left(2 \sqrt{2} x+\sqrt{2} i \partial_{\mu} \log Z_{+}\right) \\
2 \sqrt{2} x-\sqrt{2} i \partial_{\mu} \log Z_{-} & i\left(4 x^{2}+r^{2}+\mu\right)
\end{array}\right) \tilde{\Psi}
\end{aligned}
$$

where $\tilde{\Psi}=\left(\begin{array}{c}\psi_{+}(x, \mu, q) \\ \psi_{+}(x, \mu, q-1)\end{array}\right)$ and all the quantities in matrices are evaluated at $q$. Eq. (4.7) makes manifest the fact that $\psi_{+}$is the only independent function in this analysis.

A second, more interesting consequence of these identities follows from using (4.1) and the identity (2.10) to rewrite (4.5) and its charge conjugate as

$$
\left\langle B_{s}\right\rangle_{\mu, q}=\left\langle B_{-s}\right\rangle_{\mu, q+2 s}
$$

with $s= \pm 1 / 2$. Evidently, a system with closed string background labelled by $q$ and a brane $B_{s}$ is the same as a system with a closed string background labelled by $q+2 s$ with a brane $B_{-s}$ ! In other words, the distinct configurations of the system are labelled only by the value of $q+s$, and not separately by $q$ and $s$. In the next subsection, we will understand this fact in the target space language, where $q+s$ is identified as the value of the RR flux in the asymptotic weak coupling region. 


\subsection{Target space interpretation}

As discussed in the introduction, the charged FZZT branes have an open string tachyon whose value is

$$
T_{\text {open }}(\phi)=\mu_{B} e^{\frac{\phi}{2 \sqrt{2}}}=-i x e^{\frac{\phi}{2 \sqrt{2}}}
$$

The minisuperspace wavefunction $\Psi_{\text {open }}(\phi)$ shows that the brane comes in from $\phi=-\infty$ and dissolves at

$$
\phi=\phi_{0} \approx-2 \sqrt{2} \log (|x|)
$$

(Recall figure 1.) We will now argue that its charge is localized near $\phi_{0}$.

The key observation is that the charged FZZT branes are in many ways analogous to the unstable branes [30,27] of critical string theory. In that context $T_{\text {open }}=0$ is an unstable situation which can decay to one of two stable minima $T_{\text {open }}= \pm T_{0}$. The theory on these branes has a coupling to the bulk RR scalar $C$,

$$
\delta \mathcal{L}=C(\phi) \partial_{\phi} G\left(T_{\text {open }}(\phi)\right)
$$

where $G\left(T_{\text {open }}\right)$ is some function of the open string tachyon field. The charge conjugation symmetry $C \rightarrow-C, T_{\text {open }} \rightarrow-T_{\text {open }}$ shows that the function $G$ is odd and hence $G(0)=0$.

Furthermore, $G\left( \pm T_{0}\right)= \pm \frac{1}{2}$, so that a kink interpolating between the two minima carries one unit of RR charge.

Now consider our system. Here the point $T_{\text {open }}=0$ is locally stable, and the open string configuration (4.9) interpolates between $T_{\text {open }}=0$ as $\phi \rightarrow-\infty$ and $T_{\text {open }}= \pm \infty$ as $\phi \rightarrow+\infty$. Hence the FZZT brane corresponds to "half a kink" and carries charge

$$
q_{b}=\frac{1}{2} \epsilon\left(\mu_{B}\right)=\frac{1}{2} \epsilon(\operatorname{Im} x)
$$

which is localized around $\phi \approx \phi_{0}$.

Notice that the sign of the charge depends on whether $x$ is in the UHP or the LHP rather than on the value of $s$ in $B_{s}$. This immediately leads to two questions:

1. How can this be consistent with the fact that the expectation values $\psi_{ \pm}$are smooth functions of $x$ ? That is, how can the charge on the brane change as in (4.12)?

2. How is the target space charge $q_{b}$ related to the matrix model charge $s$ ?

In order to address these questions, let us examine the target space situation more closely. Consider a background with flux $q$, and introduce into this background a brane 
with charge $q_{b}$; i.e. the value of $x$ is such that $q_{b}=\frac{1}{2} \epsilon(\operatorname{Im} x)$. We start with a brane with large $|\operatorname{Im} x|$ so that it does not penetrate much into the strong coupling region $\phi \rightarrow+\infty$. Then in a first approximation, this brane does not affect the situation in the strong coupling region; in particular the flux in the strong coupling region remains the original flux,

$$
q_{\text {strong }}=q
$$

Around the point $\phi_{0}$ we have charge $q_{b}$, and correspondingly the flux in the weak coupling region $\phi \rightarrow-\infty$ is

$$
q_{w e a k}=q+q_{b}
$$

Now, as we make $x$ small and pass through $x=0$ the charge of the brane should change to $-q_{b}$. Since nothing happens at weak coupling, we expect that the flux in the weak coupling region remains $q_{\text {weak }}=q+q_{b}$. This means that the flux in the strong coupling region should change to

$$
q_{\text {strong }}=q+2 q_{b}
$$

The branes and the transition between them are depicted in figure 2 .

Returning now to the first question above, evidently the surprise is that this transition must happen smoothly. Clearly, this means that the charge $q_{b}$ and the flux $q_{\text {strong }}$ cannot jump abruptly. Instead, they must be fluctuating quantities which are in general ill defined. For large $|x|$ they freeze at the values we discuss above, but for small $|x|$ they are not well defined. This is to be contrasted with the flux at the weak coupling end $q_{\text {weak }}$, which does not change. It is specified by the boundary conditions on the system.

We interpret this result to mean that the effective volume of the strong coupling region is finite. Hence the flux there and the charge on the brane can fluctuate. On the other hand, the weak coupling region has infinite volume and hence the flux there is fixed. As $|x|$ becomes larger, the volume of the strong coupling region grows, and the fluctuations are reduced. Therefore $q_{b}$ and $q_{\text {strong }}$ freeze in this limit.

The physical picture presented here motivates the addition of the term $\pm \frac{1}{2} V_{R}|0\rangle$ in the $q=0$ boundary state (3.6). . place in it the brane created by the naive boundary state without the extra term. If this brane has large $x$, it is far in the weak-coupling region. Then we do not expect it to significantly affect the physics in the strong-coupling region, i.e. $q_{\text {strong }}=q=0$. But a

5 We thank J. Maldacena for a discussion on this point. 


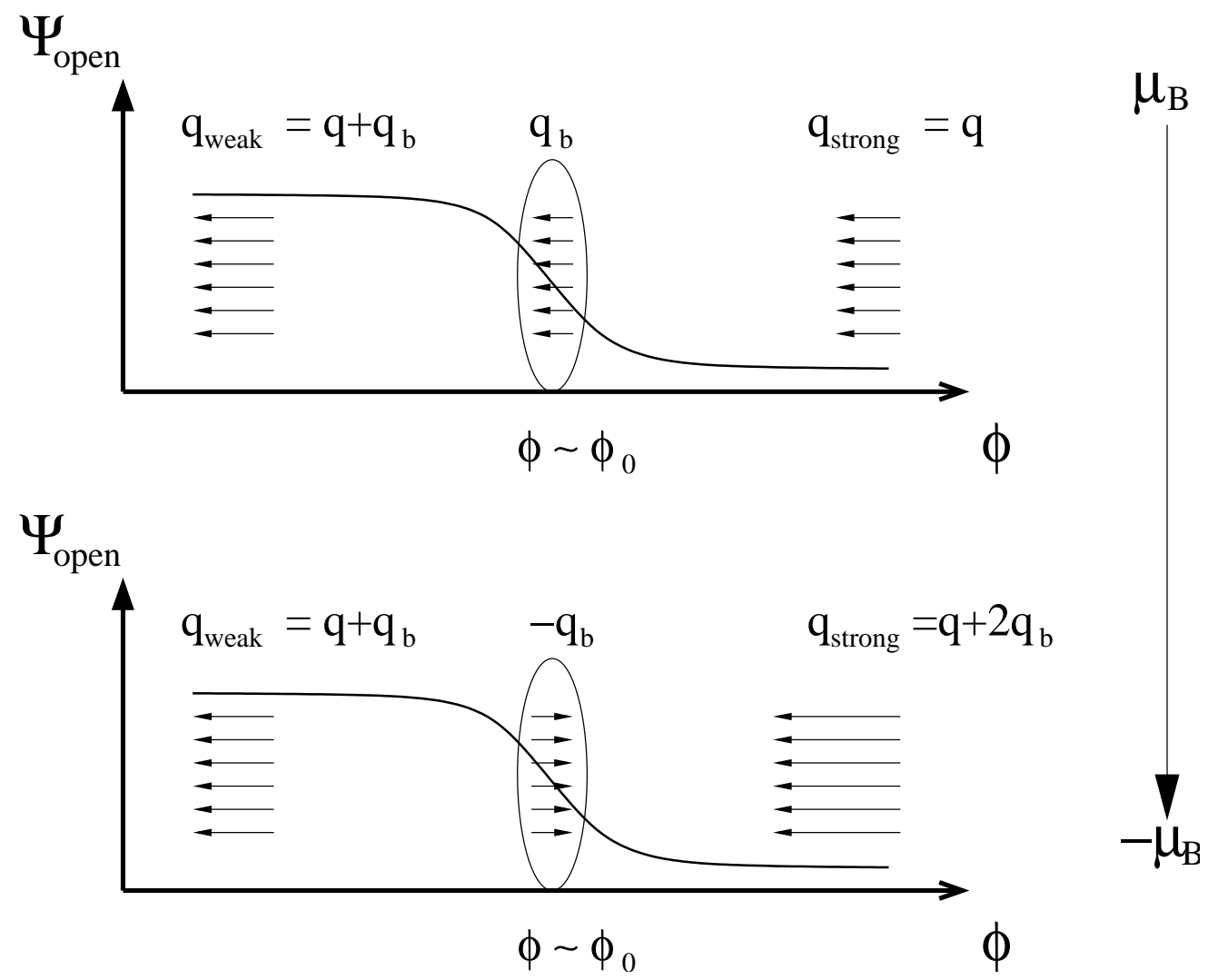

Fig. 2: The target space configuration corresponding to the charged branes. Recall that $\mu_{B}=-i x$. Semiclassically, the brane charge $q_{b}=\frac{1}{2} \epsilon\left(\mu_{B}\right)$ changes sign and the strong coupling flux $q_{\text {strong }}$ jumps by one unit as we pass from large positive to large negative $\mu_{B}$. However, the two flux configurations are smoothly connected in the exact theory.

simple calculation shows that this cannot be true if we use the naive boundary state. The flux in the weak-coupling region is measured by the one point function of the RR ground state vertex operator on the disk. Since the coefficient $\sin (2 P \sigma)$ of the RR part of the boundary state vanishes at zero momentum $P$, this one point function vanishes. Hence $q_{\text {weak }}=0$ and correspondingly, $q_{\text {strong }}=\mp \frac{1}{2}$. This contradicts our argument above that $q_{\text {strong }}=0$. Moreover, even if we allowed such flux in the strong coupling region, its value $\mp \frac{1}{2}$ would conflict with our assumption of flux quantization. These inconsistencies are cured by the added term $\pm \frac{1}{2} V_{R}|0\rangle$, which adds flux $\pm \frac{1}{2}$ everywhere, restoring $q_{\text {strong }}=0$ and leading to $q_{\text {weak }}= \pm \frac{1}{2}$.

As a check of this picture of the target space dynamics, consider again the brane identifications (4.8). The identifications were presented in section 4.1 as a consequence of the differential equations (4.2), but now we see that they fit nicely with our understanding 
of target space. According to (4.8), the same configuration can be thought of as a brane with charge $q_{b}=s$ and background flux $q_{\text {strong }}=q$, or as a brane with charge $q_{b}=-s$ and background flux $q_{\text {strong }}=q+2 s$. The brane charge and the strong coupling flux are not meaningful quantities in general. The only invariant is the net value of the flux at the weak coupling end, $q_{\text {weak }}=q+s$. This agrees precisely with our target space understanding.

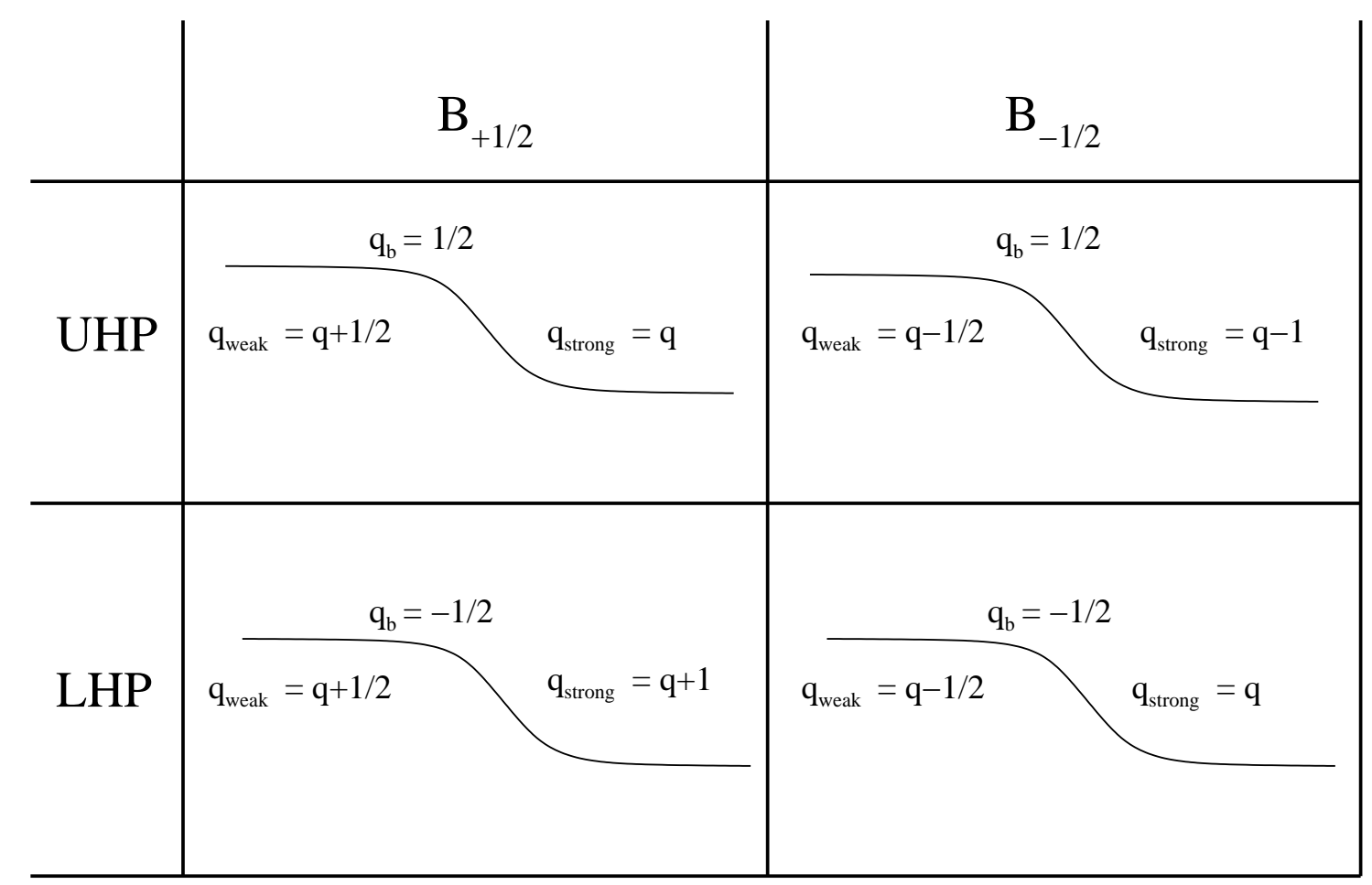

Fig. 3: The target space configuration corresponding to $B_{ \pm 1 / 2}$ for $x$ in the LHP and the UHP. The figure makes clear the fact that the label $s$ in $B_{s}$ distinguishes between different values of the weak coupling flux $q_{\text {weak }}=q+s$, and not the semiclassical brane charge $q_{b}$.

The brane identifications (4.8) also allow us to answer the second question above, namely the relation between the target space charge $q_{b}$ and the matrix model charge $s$. Clearly, the branes $B_{+1 / 2}$ and $B_{-1 / 2}$ only have their naive semiclassical charge assignments $q_{b}=1 / 2$ and $q_{b}=-1 / 2$ for $x$ in the UHP and LHP, respectively. The identifications (4.8) ensure that analytically continuing these branes to the other half plane changes the sign of $q_{b}$ and changes $q_{\text {strong }}$ by one unit of flux, in such a way that $q_{\text {weak }}$ is preserved. These four configurations are shown in figure 3.

To summarize, the various situations are labelled by $q_{\text {weak }} \in \mathbb{Z}+\frac{1}{2}$. For large $|x|$ the charge of the brane and the flux in the strong coupling region are meaningful. They are 
$q_{b}=\frac{1}{2} \epsilon(\operatorname{Im} x)$, and $q_{\text {strong }}=q_{\text {weak }}-q_{b}$. But they are not meaningful for finite $|x|$, and hence a smooth transition between these two situations is possible. It is worth mentioning that this identification of branes is reminiscent of the identification of brane charges in the context of K-theory which was discussed in [35].

\subsection{Large $|x|$ asymptotics - weak coupling limit}

We will devote the next few subsections to solving the differential equations (4.2) in various asymptotic limits. This will lead to some checks and a better understanding of the physical picture we have just described.

The simplest limit is $|x| \rightarrow \infty$ with fixed $\mu$. Since $|x|$ is large, the branes are dissolved in the weak coupling region, and therefore we expect the various fluxes and charges to be frozen. Furthermore, since $\mu$ is taken here to be of order one, the closed string background at the point the brane is dissolved is simply a linear dilaton background, and correspondingly the worldsheet theory is free.

We generalize the results of [31,32] to nonzero $q$

$$
\left(\begin{array}{c}
\psi_{+} \\
\psi_{-}
\end{array}\right) \sim\left\{\begin{array}{cc}
(-2 \sqrt{2} i x)^{q} e^{-i\left(\frac{4}{3} x^{3}+\mu x\right)}\left(\begin{array}{c}
1+\mathcal{O}(1 / x) \\
-\frac{Z_{-}(\mu, q)}{2 \sqrt{2} i x}+\mathcal{O}\left(1 / x^{2}\right)
\end{array}\right) & 0<\arg (x)<\pi \\
(2 \sqrt{2} i x)^{-q} e^{i\left(\frac{4}{3} x^{3}+\mu x\right)}\left(\begin{array}{c}
\frac{Z_{+}(\mu, q)}{2 \sqrt{2} i x}+\mathcal{O}\left(1 / x^{2}\right) \\
1+\mathcal{O}(1 / x)
\end{array}\right) & -\pi<\arg (x)<0
\end{array}\right.
$$

In the wedges $|\arg (x)|<\pi / 3$ and $|\arg (x)-\pi|<\pi / 3$ we must take the sum of the result in the UHP and LHP. Note that, although we have used the identity (4.5) to fix the overall $q$-dependent normalization of $\psi_{ \pm}$, it is still nontrivial that the $x$ and $\mu$ dependence of (4.16) respect (4.5) and (4.6).

Let us explore the expressions in more detail. For $x$ in the UHP we have $\left|\psi_{+}\right| \gg\left|\psi_{-}\right|$, while for $x$ in the LHP the opposite is true. We take this to mean that in the UHP (LHP) the naive classical picture is correct for the brane $B_{+1 / 2}\left(B_{-1 / 2}\right)$. This confirms the target space picture discussed above. We identify the power of $x$ in the asymptotic expansion (4.16) as $2 q_{b} q_{\text {strong. }}$. We will see below how this dependence can be understood from a worldsheet description. 


\subsection{Semiclassical limit}

Further insight into these phenomena arises by studying the semiclassical $g_{s} \rightarrow 0$ limit of $\psi_{ \pm}$. As noted above (2.12), this limit corresponds to $|\mu| \rightarrow \infty$. In addition, we must take $|x| \rightarrow \infty$ holding

$$
\tilde{x}=\frac{x}{\sqrt{\mu}}=\text { fixed }
$$

This way, the branes still dissolve in the weak coupling region, although now the tachyon background is nonzero there. Correspondingly, the worldsheet theory is interacting. This will allow us to explore world sheet phenomena, but with frozen background charges and fluxes. We will see that the naive semiclassical picture does not always lead to the correct answer. Instead, the correct semiclassical pictures come with different values of the flux, depending on whether we are in the UHP or the LHP. After taking this subtlety into account, we find complete agreement with worldsheet expectations.

Let us focus on the upper half plane for simplicity. The answers in the lower half plane can be easily obtained using (4.6). In the semiclassical limit, the answers depend on the sign of $\mu$. For positive $\mu$ (the one cut phase of the $0 \mathrm{~B}$ theory) we have

$$
\left(\begin{array}{c}
\psi_{+} \\
\psi_{-}
\end{array}\right) \sim e^{-i\left(\frac{4}{3} x^{3}+\mu x\right)}\left(\begin{array}{c}
Y(x, q)^{q}\left(1+\mathcal{O}\left(\mu^{-3 / 2}\right)\right) \\
Z_{-}(\mu, q) Y(x, q-1)^{q-1}\left(1+\mathcal{O}\left(\mu^{-3 / 2}\right)\right)
\end{array}\right)
$$

where

$$
Y(x, q)=-i 2 \sqrt{2}\left(x+\frac{i}{2} \epsilon(q) \sqrt{\mu}\right)
$$

and $\epsilon(q)$ was defined in (2.14). In (4.18), and later when we discuss the situation with negative $\mu$, the meaning of the correction terms like $\mathcal{O}\left(\mu^{-3 / 2}\right)$ is that they are multiplied by a function of $\tilde{x}=x / \sqrt{\mu}$.

It is easy to see that in the limit $|x| \gg|\mu| \gg 1$ these expressions are consistent with the large $|x|$ expression (4.16). Comparing with (4.16), we see that the only effect of large

$\mu$ is to shift $x$ in the prefactor by $x_{Z Z}=-\frac{i}{2} \epsilon(q) \sqrt{\mu}$. This has the following consequence: the semiclassical approximation (4.18) has a branch point at $x=x_{Z Z}$ if $q$ is not an integer. This is another example that the solutions are better behaved for integer $q$.

The physical (worldsheet) interpretation of the semiclassical limit (4.18) is illuminating. Keep in mind that in the $\mu>0$ phase, we can have charged ZZ branes. The semiclassical limit of $\psi_{+}$in the UHP is consistent with a disk amplitude

$$
D(x)=-i\left(\frac{4}{3} x^{3}+\mu x\right)
$$


This disk amplitude agrees with the results in section 3 and in [13, 15. The asymptotics of $\psi_{+}$also imply that the annulus between the FZZT brane and itself is zero,

$$
Z_{\text {annulus }}(x, x)=0
$$

while the log of the prefactor

$$
\tilde{Z}_{\text {annulus }}(x, q)=q \log Y(x, q)
$$

can be interpreted as due to an annulus diagram with one end on the FZZT brane and the other on $|q|$ ZZ branes. More precisely, note that $Y(x, q)$ can be written as

$$
\begin{aligned}
& Y=-i 2 \sqrt{2}\left(x-x_{Z Z}(q)\right) \\
& x_{Z Z}(q)=-\frac{i}{2} \epsilon(q) \sqrt{\mu}
\end{aligned}
$$

where the value of $x_{Z Z}$ is the position of the ZZ branes on the semiclassical Riemann surface [13,15]. Notice that all of the $|q|$ background ZZ branes are located at just one of the two singularities of the surface, depending on the sign of $q$. We will return to this point below, in section 4.6. The formula (4.22) is analogous to the formula for the FZZT-ZZ annulus in the bosonic string as derived in [21]. It is interesting that the annulus (4.23) depends on $q$ rather than $|q|$. This indicates that it involves the exchange of the RR Ishibashi states in the boundary state (3.1). (Of course, the NS Ishibashi states can contribute as well.)

Consider now $\psi_{-}$. The general target space picture discussed above implies that $\psi_{-}$ in the UHP describes a configuration of a brane with charge $q_{b}=\frac{1}{2}$ and strong-coupling flux $q_{\text {strong }}=q-1$ (see figure 3 ). Thus the disk amplitude and annulus between the brane and itself are the same as for $\psi_{+}$. Meanwhile, the $q$ dependence in this phase comes from the interaction of the brane and $|q-1|$ charged ZZ branes located at $x_{Z Z}(q-1)$. As in our large $x$ expression (4.16), the power of $Y$ is given by $2 q_{b} q_{\text {strong }}$. It is different from $\psi_{+}$ because $q_{\text {strong }}$ is different, but it can still be interpreted as an annulus between an FZZT brane and $\left|q_{\text {strong }}\right| \mathrm{ZZ}$ branes. The prefactor $Z_{-}(\mu, q)$ leads to exponential enhancement for positive $q$ and exponential suppression for negative $q$. This is consistent with the change in the number of ZZ branes. It is straightforward to extend this discussion to the LHP.

It is also instructive to understand in this semiclassical language what happens to the system as we bring the brane $B_{-1 / 2}$ from the LHP to the UHP. Suppose we start with $\operatorname{Im} x$ large and negative, where the system is described by strong-coupling flux $q$ and a brane 
with charge $-1 / 2$. Increasing $x$ through $x=0$ and out to large positive $\operatorname{Im} x$ corresponds to bringing the brane into the strong coupling region and back out again. Then we find that the system is described by strong coupling flux $q-1$ and a brane with charge $+1 / 2$. For positive $q$ we see a transition, whereby the brane picks up a $Z Z$ brane from the strong coupling region and carries it back out to weak coupling. For negative $q$ the brane leaves behind an anti-ZZ brane and then returns.

Now let us consider the semiclassical limit for negative $\mu$ (the two cut phase of the 0B theory). Here we find

$$
\left(\begin{array}{c}
\psi_{+} \\
\psi_{-}
\end{array}\right) \sim \frac{e^{-i \frac{4}{3}\left(x^{2}+\mu / 2\right)^{3 / 2}}}{\sqrt{2}\left(x^{2}+\mu / 2\right)^{1 / 4}}\left(\begin{array}{c}
(-i \sqrt{2})^{q}\left(x+\sqrt{x^{2}+\mu / 2}\right)^{\frac{1}{2}+q}\left(1+\mathcal{O}\left(\mu^{-3 / 2}\right)\right) \\
(-\mu)^{-q+\frac{1}{2}}(-i \sqrt{2})^{q-1}\left(x+\sqrt{x^{2}+\mu / 2}\right)^{-\frac{1}{2}+q}\left(1+\mathcal{O}\left(\mu^{-3 / 2}\right)\right)
\end{array}\right)
$$

The substitution $x=i \sqrt{-\frac{\mu}{2}} \sinh \sigma$ (see section 3 ) simplifies this to

$$
\left(\begin{array}{c}
\psi_{+} \\
\psi_{-}
\end{array}\right) \sim \frac{e^{-\frac{4}{3}(-\mu / 2)^{3 / 2} \cosh ^{3} \sigma}}{\sqrt{2 \cosh (\sigma)}}\left(\begin{array}{c}
(-\mu)^{q / 2} e^{\left(q+\frac{1}{2}\right) \sigma}\left(1+\mathcal{O}\left(\mu^{-3 / 2}\right)\right) \\
(-\mu)^{-q / 2} e^{\left(q-\frac{1}{2}\right) \sigma}\left(1+\mathcal{O}\left(\mu^{-3 / 2}\right)\right)
\end{array}\right)
$$

This parametrization makes it manifest that these asymptotics are valid for $x$ in both the UHP and the LHP.

The physical interpretation of these asymptotics is as follows. The leading effect for both branes arises from a disk amplitude

$$
D(x)=-i \frac{4}{3}\left(x^{2}+\mu / 2\right)^{3 / 2}=-\frac{4}{3}(-\mu / 2)^{3 / 2} \cosh ^{3} \sigma
$$

This value and its derivative $y=\partial_{x} D(x)=-4 i x \sqrt{x^{2}+\mu / 2}=-i \mu \sinh (2 \sigma)$ are as in section 3 and in [13, 15]. The corrections to this result have not yet been computed using worldsheet methods, but we can read them off from (4.25). Here, we will see that the new term $\pm \frac{1}{2} V_{R}|0\rangle$ in (3.6) is important. Let us start with $\psi_{+}$. We interpret the log of the $q$-independent prefactor in (4.25)

$$
Z_{\text {annulus }}(\sigma, \sigma)=-\log (2 \cosh \sigma)+\sigma
$$

as the result of an annulus diagram whose two ends are on the FZZT brane (3.6). We can understand better the effect of the new term $\pm \frac{1}{2} V_{R}|0\rangle$ by decomposing (4.27) into three terms

$$
Z_{\text {annulus }}(\sigma, \sigma)=\partial_{q} D^{\text {naive }}(\sigma)-\frac{1}{4} \partial_{q}^{2} F+Z_{\text {annulus }}^{\text {naive }}(\sigma, \sigma)
$$


where the derivatives $\partial_{q}$ are evaluated at $q=0$. These terms have the following interpretations:

1. The first term arises from the disk one point function of the RR vertex operator with the "naive" boundary state, by which we mean (3.6) without the extra $\pm \frac{1}{2} V_{R}|0\rangle$ term:

$$
\partial_{q} D^{\text {naive }}(\sigma)=\left\langle V_{R} \mid \sigma, \eta=-1\right\rangle_{+}^{\text {naive }}=\sigma
$$

This answer can be found by expressing the $\phi$ dependence of the vertex operator as $\phi e^{Q \phi / 2}=\lim _{k \rightarrow 0} \partial_{k} e^{(k+Q / 2) \phi}$, and reading off the coefficient of the RR Ishibashi state with $i P=-k \sqrt{2}$. (The overall normalization is fixed by comparing with the matrix model answer (4.25).) As a check, note that it is odd under $\sigma \rightarrow-\sigma$, as expected since the $\mathrm{R}$ component of (3.6) is multiplied by $\sin (2 P \sigma)$.

2. The second term in (4.28) arises from two insertions of $V_{R}$ on the sphere

$$
-\partial_{q}^{2} F=\left\langle V_{R} V_{R}\right\rangle=\log (-\mu)
$$

where we have used the semiclassical expansion (2.17).

3. Finally, the third term in (4.28) arises from the annulus with two ends on the naive boundary state:

$$
Z_{\text {annulus }}^{\text {naive }}(\sigma, \sigma)=-\log (\cosh \sigma)-\frac{1}{4} \log (-\mu)
$$

As a check, note that this is invariant under $\sigma \rightarrow-\sigma$ as expected from the form of the boundary state. We did not calculate this using the worldsheet, but simply matched with the answer (4.27), assuming the decomposition (4.28). In particular, the term proportional to $\log (-\mu)$ was designed to cancel the similar term in (4.30).

Armed with these terms, we can now understand the $q$ dependence of $\psi_{+}$in (4.25). Recall that there are no ZZ branes for negative $\mu$, and instead $q$ represents the coefficient of the RR vertex operator in the worldsheet theory. Thus the log of the $q$-dependent prefactor in (4.25) arises from the one-point function of the $R R$ vertex operator in the boundary state (3.6). (Insertions of more vertex operators are higher order in the semiclassical expansion, and contributions without the boundary state (3.6) are part of the closed string partition function.) This leads to

$$
q \partial_{q} D(\sigma)=q\left(\partial_{q} D^{\text {naive }}(\sigma)-\frac{1}{2} \partial_{q}^{2} F\right)=q\left(\sigma+\frac{1}{2} \log (-\mu)\right)
$$


where again $\partial_{q}$ is evaluated at $q=0$. The first term arises from a one point function on a disk with the naive boundary state and the second from a sphere with two insertions of $V_{R}$ - one of them from the background flux and the other from the vertex operator in the boundary state (3.6). The fact that (4.32) agrees with (4.25) is a nontrivial check of our corrected boundary state (3.6).

Having understood the worldsheet interpretation $\psi_{+}$, now let us turn to $\psi_{-}$. From the form of the boundary state, the leading order term $D(x)$ and the $q$-independent correction $Z_{\text {annulus }}(\sigma, \sigma)$ in $\psi_{-}$should be the same as for $\psi_{+}$. But the $q$-dependent correction (4.32) should be different, because this brane is put in a background flux $q_{\text {strong }}=q-1$. It has the same charge $q_{b}=+\frac{1}{2}$, so the insertion of the RR vertex operator on the disk, which is proportional to $q_{b} q_{\text {strong }}$, gives $(q-1) \partial_{q} D(\sigma)=(q-1)\left(\sigma+\frac{1}{2} \log (-\mu)\right)$. Finally, we have to account for the crucial factor of $Z_{-} \approx(-\mu)^{-q+\frac{1}{2}}$ which corrects the closed-string free energy as we change $q$ to $q-1$. Putting all this together, we find that the worldsheet prediction for the leading-order correction to $\psi_{-}$agrees precisely with the asymptotics of $\psi_{-}$in (4.25). Of course, this was guaranteed to work, given the identity (4.5). Even so, it is still nice to see how it all fits together with the worldsheet description.

\section{5. $x \sim 1$, large $|\mu|$ asymptotics}

Another interesting limit is large $|\mu|$ but with $x$ of order one. In this limit, the theory is still semiclassical, but the worldsheet is strongly coupled and the branes penetrate deep into the strong-coupling region. The flux and brane charge are always fluctuating, so the analytic continuation of $x$ between the UHP and the LHP must be manifestly smooth. Correspondingly, the effects of Stokes' phenomenon, as well as the charge conjugation symmetry (4.6), will be explicit in this limit.

For $\mu \rightarrow+\infty, x \sim 1$, we find

$$
\begin{array}{r}
\left(\begin{array}{c}
\psi_{+} \\
\psi_{-}
\end{array}\right) \sim e^{-i\left(\frac{4}{3} x^{3}+\mu x\right)}\left(\begin{array}{c}
(\epsilon(q) \sqrt{2 \mu})^{q}\left(1+\mathcal{O}\left(\mu^{-1 / 2}\right)\right) \\
(\epsilon(q-1) \sqrt{2 \mu})^{q-1} Z_{-}(\mu, q)\left(1+\mathcal{O}\left(\mu^{-1 / 2}\right)\right)
\end{array}\right) \\
+e^{i\left(\frac{4}{3} x^{3}+\mu x\right)}\left(\begin{array}{c}
(-\epsilon(q+1) \sqrt{2 \mu})^{-q-1} Z_{+}(\mu, q)\left(1+\mathcal{O}\left(\mu^{-1 / 2}\right)\right) \\
(-\epsilon(q) \sqrt{2 \mu})^{-q}\left(1+\mathcal{O}\left(\mu^{-1 / 2}\right)\right)
\end{array}\right)
\end{array}
$$

As expected, this formula is smooth and is valid for $x$ in both the UHP and the LHP. Along the real $x$ axis the exact answer has an anti-Stokes' line, which becomes a branch cut discontinuity in the classical limit. On the anti-Stokes' line, the two terms in (4.33) are equally important. Moving $x$ into the UHP (LHP), the first (second) term dominates 
as $|x| \rightarrow \infty$, as needed for consistency with the semiclassical limit (4.18). The relative coefficient between the two terms is determined by the charge conjugation symmetry (4.6).

It is interesting to note that a similar phenomenon happens as $\mu \rightarrow+\infty$, due to the factors of $Z_{ \pm}$in (4.33). (Recall the asymptotic expansions in section 2.2.) That is, each of the functions $\psi_{ \pm}$is dominated at large $\mu$ by only one of the two exponentials in (4.33). The other one is exponentially suppressed in the $\mu \rightarrow+\infty$ limit. Which exponential dominates in $\psi_{ \pm}$depends on $q$. For $q=0$ we have $\psi_{ \pm} \sim e^{\mp i\left(\frac{4}{3} x^{3}+\mu x\right)}$. This means that in this case the fixed charge branes are approximately "left-moving" and "right-moving" waves. Meanwhile, for $q \neq 0$ we have $\psi_{ \pm} \sim e^{-\epsilon(q) i\left(\frac{4}{3} x^{3}+\mu x\right)}$. Here the fixed charge branes are either both "left-moving" or both "right-moving" waves.

In the limit $\mu \rightarrow-\infty, x \sim 1$, we find

$$
\left(\begin{array}{c}
\psi_{+} \\
\psi_{-}
\end{array}\right) \sim \frac{1}{\sqrt{2}} e^{-\frac{\sqrt{2}}{3}(-\mu)^{3 / 2}+\sqrt{2} x^{2}(-\mu)^{1 / 2}}\left(\begin{array}{c}
(-\mu)^{q / 2}\left(1+\mathcal{O}\left(\mu^{-1 / 2}\right)\right) \\
(-\mu)^{-q / 2}\left(1+\mathcal{O}\left(\mu^{-1 / 2}\right)\right)
\end{array}\right)
$$

for $x$ in both the UHP and the LHP. One can check that (4.34) agrees exactly with the $x \rightarrow 0$ limit of the semiclassical asymptotics 4.24)-(4.25). In contrast with the positive $\mu$ limit (4.33), there is only one term (and no anti-Stokes' line) in the negative $\mu$ limit. This agrees with the fact that there is no branch cut near $x=0$ in the semiclassical answer (4.24). One can also see that (4.34) satisfies the charge conjugation symmetry. Since (4.34) matches smoothly onto the semiclassical answer which decays as $x \rightarrow \pm i \infty$, this is evidence that the physical boundary conditions on $\psi_{ \pm}$are consistent for every value of $q$.

\subsection{A large q limit}

Finally, let us consider a modified version of the semiclassical limit in section 4.4, where we not only take $|x|$ and $|\mu|$ to infinity keeping $\tilde{x}$ in (4.17) fixed, but also send $|q| \rightarrow \infty$ keeping

$$
\tilde{q}=\frac{q}{\mu^{3 / 2}}=\text { fixed }
$$

This limit was studied in [13], where it was shown to smooth out the Gross-Witten phase transition even in the classical limit.

Rather than extract the detailed asymptotics of $\psi_{ \pm}$as in the preceding subsections, let us just focus on the WKB exponent for simplicity. This can be efficiently extracted from the differential equations (4.2) as follows. Take the matrix $A_{x}$ in (4.2), and drop all 
derivatives of $r(\mu, q)$. But keep the derivatives of $\beta(\mu, q)-$ according to (2.2), these are proportional to $q \sim \frac{1}{g_{s}}$. The eigenvalues of this matrix are given by $\pm y(x)$ where

$$
y^{2}=-16\left(x-\frac{i q}{2 r^{2}}\right)^{2}\left(x-\frac{r}{\sqrt{2}}+\frac{i q}{2 r^{2}}\right)\left(x+\frac{r}{\sqrt{2}}+\frac{i q}{2 r^{2}}\right)
$$

(compare with the discussion of the algebraic curve in section 3) and $r$ satisfies the genus zero version of the string equation (2.3), i.e.

$$
-\mu r-r^{3}+\frac{q^{2}}{r^{3}}=0
$$

The WKB exponents (or at least their $x$-dependent parts) are then

$$
D(x)=\int^{x} y\left(x^{\prime}\right) d x^{\prime}
$$

where the sign of $y$ is determined by the boundary conditions on $\psi_{ \pm}$. As a check of these formulas, note that in the small $\tilde{q}$ limit, (4.38) reduces to

$$
D(x)= \begin{cases}-i\left(\frac{4}{3} x^{3}+\mu x\right)+q \log \left(x+\frac{i}{2} \epsilon(q) \sqrt{\mu}\right)+\mathcal{O}\left(\mu^{-3 / 2}\right) & \mu>0 \\ -i \frac{4}{3}\left(x^{2}+\mu / 2\right)^{3 / 2}+q \log \left(x+\sqrt{x^{2}+\mu / 2}\right)+\mathcal{O}\left(\mu^{-3 / 2}\right) & \mu<0\end{cases}
$$

Here we have assumed $x \in \mathrm{UHP}$, and we have imposed the proper boundary conditions. (4.39) agrees with the large $q, x$ dependent part of the semiclassical expansions (4.18) and (4.24), showing that the various limits are consistent with one another.

The algebraic curve $y(x)$ in (4.36) defines a Riemann surface that is a double cover of the complex $x$ plane. It generalizes the $q=0$ surface of the $\eta=-1$ FZZT brane reviewed in section 3. In [15], it was shown how this surface unifies geometrically many features of minimal string theory. So let us examine its $q$-deformed generalization in more detail. According to (4.37) (see also (2.13) and (2.15) ) $r$ is nonzero when $q$ is nonzero. Then the curve (4.36) always has exactly one singularity at

$$
x=\frac{i q}{2 r^{2}}
$$

for either sign of $\mu$. For $\mu>0$, the $q=0$ curve (3.5) has two singularities at $x= \pm \frac{i \sqrt{\mu}}{2}$, so nonzero $q$ has the effect of opening up one of these singularities while shifting continuously the other. For $\mu<0$, the $q=0$ curve (3.9) has only one singularity at $x=0$, and one can check using (2.13) that nonzero $q$ continuously shifts the location of this singularity without opening it up. 
Physically, changing $q$ corresponds to adding charged ZZ branes to the system for $\mu>0$, and it corresponds to insertions of the closed-string $\mathrm{RR}$ vertex operator for $\mu<0$. Therefore, our analysis of the $q$-deformed curve is consistent with the general idea in [21] that adding background ZZ branes opens up singularities, while deforming by closed string operators preserves the singularities.

Let us take a closer look at the $q$ deformation for $\mu>0$. When $q$ is zero, the singularities are in the upper and lower half plane, symmetric under $x \rightarrow-x$. This symmetry is broken by nonzero $q$. Turning on $q$ splits one of the singularities depending on the sign of $q$. According to (4.40), the split singularity is in the LHP (UHP) for $q>0$ $(q<0)$. We identify the sign of $q$ (and which singularity is split) with the charge of the $(1,1)$ ZZ brane that exists in the phase. Note that we already saw a hint of this symmetry breaking in our study of the annulus amplitude (4.22).

Another interesting feature of the $q$ deformed curve can be seen by expanding $y(x)$ around $x=\infty$. From (4.36), we find (for both signs of $\mu$ )

$$
y=-i\left(4 x^{2}+\mu\right)+\frac{q}{x}+\ldots
$$

Therefore, we can characterize the effect of $q$ as a deformation of the curve which introduces a pole at infinity. Equivalently, we can say that the Riemann surface of the charged brane has a puncture at $x=\infty$. Later, when we study the surface of the neutral brane, we will see that this feature at infinity has an important role to play.

\section{The neutral branes}

\subsection{The differential equations}

In addition to charged branes, our system also has a stable neutral brane $B_{0}$ in its spectrum, corresponding to the $\eta=+1$ FZZT brane in the worldsheet description. This brane is most natural in the 0A language. Using the dual matrix model, one finds that the partition function

$$
\psi_{0}=\psi_{0}(x, \mu, q)=\frac{\left\langle B_{0}(x)\right\rangle_{\mu, q}}{\mathcal{Z}(\mu, q)}
$$

satisfies the following differential equations:

$$
\begin{aligned}
& \partial_{\mu}^{2} \psi_{0}=\left(r^{2}+\mu-2 x^{2}\right) \psi_{0} \\
& x \partial_{x} \psi_{0}=\left(r r^{\prime}-|q|\right) \psi_{0}-\left(r^{2}+4 x^{2}\right) \partial_{\mu} \psi_{0}
\end{aligned}
$$


Again, the derivation of (5.2) is left to appendix A.2. The structure of these equations is similar to the corresponding equations in the bosonic string [36,37]. As for the charged branes, we can list several consistency checks. First, the equations are clearly invariant under the shift symmetry of $\beta$ (recall the discussion below (2.6)), so $\psi_{0}$ indeed has zero charge. Second, one can check that the compatibility of the two equations in (5.2) is equivalent to the string equation (2.3). Finally, one can show using the results of [38 that there exists a solution to (5.2) that is smooth in the entire complex $x$ plane.

Given that $\psi_{0}$ is the double-scaling limit of the orthonormal wavefunction $\psi_{N}=$ $e^{-V\left(x^{2}\right) / 2} P_{N}\left(x^{2}\right)$ of the complex matrix model (see appendix A.3), it must have the following symmetries:

$$
\psi_{0}(x, \mu, q)=\psi_{0}(-x, \mu, q), \quad \psi_{0}(x, \mu, q)=\psi_{0}(x, \mu,-q)
$$

In addition, we expect that $\psi_{0} \rightarrow 0$ exponentially as $i x \rightarrow \pm \infty$, since the matrix model potential goes to infinity there.

As for the charged brane, there exists an identity which relates branes of different $q$ (see e.g. 39):

$$
\psi_{0}(x, \mu, q+1)=\frac{1}{x^{2}}\left(\partial_{\mu} \psi_{0}(x, \mu, q)-\psi_{0}(x, \mu, q) \partial_{\mu} \log Z_{+}(\mu, q)\right)
$$

Here we have assumed $q \geq 0$ for simplicity; the result for $q<0$ can be obtained using the charge conjugation symmetry (5.3). It is straightforward to check explicitly that (5.4) satisfies the differential equations (5.2) with $q \rightarrow q+1$. In appendix A.3, we also offer a microscopic derivation starting from the complex matrix model. Using the definition (5.1) of $\psi_{0}$, we can also write (5.4) as

$$
\left\langle B_{0}(x)\right\rangle_{\mu, q+1}=\frac{1}{x^{2} \mathcal{Z}(\mu, q)}\left(\left\langle B_{0}(0)\right\rangle_{\mu, q} \partial_{\mu}\left\langle B_{0}(x)\right\rangle_{\mu, q}-\partial_{\mu}\left\langle B_{0}(0)\right\rangle_{\mu, q}\left\langle B_{0}(x)\right\rangle_{\mu, q}\right)
$$

Here we have also used $\psi_{0}(x=0, \mu, q)=Z_{+}(\mu, q)$ for $q>0$, a fact that we will discuss further in the next subsection and derive in appendix A.3. There, we will also interpret (5.5) as the statement that for the neutral brane, changing $q \rightarrow q+1$ is equivalent to inserting $B_{0}$ at $x=0$. 


\subsection{Asymptotics}

The various asymptotic limits of the neutral brane are much simpler than than those of the charged branes.

1. Large $x$, everything else held fixed. Then

$$
\psi_{0} \sim \frac{1}{2 \sqrt{\pi}}\left(-\frac{i x}{\sqrt{2}}\right)^{-|q|-1 / 2} e^{-i \sqrt{2}\left(\frac{4}{3} x^{3}-\mu x\right)}(1+\mathcal{O}(1 / x))
$$

in the UHP, and similarly in the LHP using (5.3). Note that these simple asymptotics are valid for any sign of $\mu$, and for both $q$ zero and nonzero.

2. Semiclassical limit, $\mu<0$. The string equation is solved by $r=r(\mu, q)$ given in (2.13), and we have

$$
\psi_{0} \sim \frac{1}{2 \sqrt{\pi}}\left(-\frac{i x}{\sqrt{2}}\right)^{-|q|-1 / 2} e^{-i \sqrt{2}\left(\frac{4}{3} x^{3}-\mu x\right)}\left(1+\mathcal{O}\left(\mu^{-3 / 2}\right)\right)
$$

in the UHP. This answer obviously agrees with (5.6) at $|x| \rightarrow \infty$. The overall factor of $x^{-|q|}$ can be removed by redefining $\psi_{0}$. If we do that, it will change the term $|q|$ in (5.2). Our choice of (5.2) and $\psi_{0}$ guarantees that $\psi_{0}$ is entire in $x$ and for all (not necessarily integer) $q$. Apart from this overall factor, we see from (5.7) that $q$ does not contribute to either the disk or the annulus amplitude in this phase. We can understand this as follows. In this phase, we have flux but no charged ZZ branes. Since the neutral brane has no RR Ishibashi component in its boundary state, the disk with a single insertion of the $\mathrm{RR}$ vertex operator must be zero. Thus the leading order semiclassical limit is necessarily $q$-independent. The first contribution will come at $\mathcal{O}\left(q^{2}\right)$, from the two-point function of the $\mathrm{RR}$ vertex operator.

3. Semiclassical limit, $\mu>0$. Here $r(\mu, q)$ is given by (2.15) for $q \neq 0$ and by (2.12) for $q=0$. However, we find a unified formula for the asymptotics

$$
\psi_{0} \sim \frac{1}{\sqrt{2 \pi}}\left(\mu-2 x^{2}\right)^{-1 / 4}\left(\frac{\sqrt{\mu}+\sqrt{\mu-2 x^{2}}}{2}\right)^{-|q|} e^{-\frac{4}{3} \sqrt{2}\left(\frac{\mu}{2}-x^{2}\right)^{3 / 2}}\left(1+\mathcal{O}\left(\mu^{-3 / 2}\right)\right)
$$

in the UHP. The answer in the LHP is similar, using (5.3). Again, one can check that this correctly reduces to (5.6) as $x \rightarrow+i \infty$. The fact that $\psi_{0}$ depends only on $|q|$ implies that its interaction with the charged ZZ branes that exist in this phase 
proceeds solely through the exchange of NSNS states. This is consistent with the boundary state for the neutral brane, which does not have a RR Ishibashi component.

4. Small $x$, everything else held fixed. Then we find

$$
\psi_{0} \sim Z_{+}(\mu,|q|)\left(1+\mathcal{O}\left(x^{2}\right)\right)
$$

where $Z_{ \pm}=r e^{\mp \beta}$ as above. The answer clearly respects the symmetries (5.3). It agrees with the small $x$ limit of the semiclassical limit for $\mu>0$, but not for $\mu<0$. Of course, the differential equations (5.2) only fix $\psi_{0}$ up to an overall $q$-dependent factor. More work is required to show that $\psi_{0}$ reduces to precisely $Z_{+}(\mu,|q|)$ at $x=0$ with no additional $q$-dependent normalization factor. We show this in appendix A.3.

\subsection{A large $q$ limit}

Just as for the charged branes, it is interesting to consider a modified semiclassical limit, where we take $q$ to infinity holding fixed $\tilde{q}=q / \mu^{3 / 2}$. To extract the WKB exponent from the differential equations (5.2), we define the column vector $\Psi_{0}=\left(\begin{array}{c}\psi_{0} \\ \partial_{\mu} \psi_{0}\end{array}\right)$. The action of $\partial_{x}$ on $\Psi_{0}$ is a $2 \times 2$ matrix

$$
\partial_{x} \Psi_{0}=\frac{1}{x}\left(\begin{array}{cc}
r r^{\prime}-|q| & -4 x^{2}-r^{2} \\
8 x^{4}-2 x^{2}\left(2 \mu+r^{2}\right)+\left(r^{\prime 2}-\frac{q^{2}}{r^{2}}\right) & -r r^{\prime}-|q|
\end{array}\right) \Psi
$$

After dropping the $\mu$ derivatives, the eigenvalues of this matrix become $-\frac{|q|}{x} \pm y(x)$ with $y(x)$ satisfying

$$
y^{2}=-\frac{\left(2 x^{2}-r^{2}-\mu\right)\left(4 x^{2}+r^{2}\right)^{2}}{x^{2}}
$$

Here $r$ again satisfies the genus zero string equation 4.37). The WKB exponent of $\psi_{0}$ is then

$$
D(x)=-|q| \log x+\int^{x} y\left(x^{\prime}\right) d x^{\prime}
$$

As a consistency check, we can compare the small $q$ limit of (5.12) with the large $q$ limit of the semiclassical limit above. From (5.12), we find

$$
D(x)= \begin{cases}-\frac{4}{3} \sqrt{2}\left(\frac{\mu}{2}-x^{2}\right)^{3 / 2}-|q| \log \left(\sqrt{\frac{\mu}{2}}+\sqrt{\frac{\mu}{2}-x^{2}}\right)+\mathcal{O}\left(q^{2}\right) & \mu>0 \\ -i \sqrt{2}\left(\frac{4}{3} x^{3}-\mu x\right)-|q| \log x+\mathcal{O}\left(q^{2}\right) & \mu<0\end{cases}
$$

which agrees precisely with the asymptotics (5.7)-(5.8).

The algebraic curve (5.11) generalizes to nonzero $q$ the curve of the $\eta=+1$ FZZT brane reviewed in section 3 and studied in detail in [15]. For nonzero $q$, the curve (5.11) 
always has two singularities at $x= \pm i r / 2$ and a pole at $x=0$ with residue $\pm q$. For $\mu<0$, the two singularities at $x= \pm i r / 2$ are continuously connected to the two singularities at $x= \pm i \sqrt{-\mu} / 2$ of the $q=0$ surface (3.9). So the main effect of turning on $q$ is to create the pole at $x=0$. For $\mu>0$, the singularity at $x=0$ of the $q=0$ surface (3.5) is split to two singularities at $x= \pm i r / 2$, and a pole at $x=0$ is created. The $q$ deformation in this phase comes from charged ZZ branes; specifically, we expect it to be the result of the annulus diagram between the $\eta=+1$ FZZT and the $\eta=-1$ ZZ brane.

As we noted in section 3 , the $q=0$ curves for the two signs of $\eta$ are essentially the same, related by $\mu \rightarrow-\mu$. However, comparing (4.36) and (5.11) shows that they are quite different for nonzero $q$. There are two important differences between them. First, for the neutral brane $y(x)$ (5.11) has a pole with residue $\pm q$ at $x=0$, while for the charged brane (4.36) $y(x)$ is smooth at every finite $x$. Second, the charged brane curve has such a pole at infinity (4.41), while the curve of the neutral brane does not have a pole there:

$$
y=-i \sqrt{2}\left(4 x^{2}-\mu\right)+\mathcal{O}\left(\frac{1}{x^{2}}\right)
$$

Equivalently, we can say that the surface for the charged brane has a puncture at $x=\infty$, while the surface for the neutral brane has a puncture at $x=0$. These differences serve to distinguish between the surfaces of the charged and neutral branes, which would otherwise be related by $\mu \rightarrow-\mu$.

\section{Acknowledgments:}

We would like to thank D. Gaiotto, S. Hellerman, A. Its, V. Kazakov, I. Klebanov, J. Maldacena, G. Moore and K. Okuyama for useful discussions. NS thanks his friends and colleagues at the Racah Institute of Physics for hospitality during the completion of this work. The research of NS is supported in part by DOE grant DE-FG02-90ER40542. The research of DS is supported in part by an NSF Graduate Research Fellowship and by NSF grant PHY-0243680. Any opinions, findings, and conclusions or recommendations expressed in this material are those of the author(s) and do not necessarily reflect the views of the National Science Foundation. 


\section{Appendix A. The integrable hierarchies of the minimal superstring}

\section{A.1. Type $O B$}

The matrix model dual to $0 \mathrm{~B}$ minimal string theory is [13]:

$$
\mathcal{Z}_{N}=\int d M e^{-\operatorname{Tr} V(M)}
$$

where $M$ is an $N \times N$ Hermitian matrix, and $V(M)$ is polynomial in $M$. The model describing our system has $V(M)=-M^{2}+g M^{4}$; the continuum "double-scaling" limit consists of taking $N \rightarrow \infty$ while simultaneously tuning $g$ to some critical value. In this limit, $\mathcal{Z}_{N}$ becomes the bulk partition function $\mathcal{Z}(\mu, q)$, and the eigenvalues of $M$ become distributed along finite "cuts" on the real axis.

Recall that in the bosonic string, the FZZT partition function $\psi_{b o s}(x)$ is obtained from double-scaling the orthonormal wavefunction of the matrix model,

$$
\psi_{\text {bos }}(x)=\lim _{N \rightarrow \infty} \psi_{N}(x)
$$

which at finite $N$ is the expectation value of the determinant operator $\operatorname{det}(x-M)$,

$$
\psi_{N}(x)=\frac{1}{\sqrt{h_{N}}} e^{-V(x) / 2}\langle\operatorname{det}(x-M)\rangle
$$

Here the story is similar, except that the even and odd orthonormal wavefunctions have different scaling limits. That is, if we take $N$ to be even, then we study the two functions $\psi_{\text {even }}(x)$ and $\psi_{\text {odd }}(x)$ via

$$
\begin{aligned}
& \psi_{\text {even }}(x)=\lim _{N \rightarrow \infty} \psi_{N}(x) \\
& \psi_{\text {odd }}(x)=\lim _{N \rightarrow \infty} \psi_{N-1}(x)
\end{aligned}
$$

$\psi_{N}(x)$ is still related to the determinant operator through (A.3), while $\psi_{N-1}(x)$ is related to the inverse determinant through

$$
\frac{1}{\sqrt{h_{N-1}}} e^{V(x) / 2}\left\langle\frac{1}{\operatorname{det}(x-M)}\right\rangle=\int_{-\infty}^{\infty} d \lambda e^{(V(x)-V(\lambda)) / 2} \frac{\psi_{N-1}(\lambda)}{x-\lambda}
$$

$\psi_{N-1}$ can be extracted from this expectation value by subtracting the value of this function above and below the real axis. This makes it clear that both $\psi_{\text {even }}$ and $\psi_{\text {odd }}$ are physical observables of the matrix model. 
Now, we claim that the partition functions of the charged FZZT branes correspond to the linear combinations

$$
\psi_{ \pm}(x)=\psi_{\text {even }}(x) \pm i \psi_{\text {odd }}(x)
$$

To see this, we need to quickly review the Zakharov-Shabat integrable hierarchy which underlies $0 \mathrm{~B}$ minimal superstring theory [2-7]. It can be formulated in terms of $2 \times 2$ matrix differential operators 8

$$
Q=\left(\begin{array}{cc}
i \partial_{\tau} & -\frac{i Z_{+}}{\sqrt{2}} \\
\frac{i Z_{-}}{\sqrt{2}} & -i \partial_{\tau}
\end{array}\right)
$$

and

$$
P=\sum_{j \geq 0}\left(t_{2 j+1}\left(-Q^{2}\right)_{+}^{j+1 / 2}+t_{2 j}\left(Q\left(-Q^{2}\right)^{j-1 / 2}\right)_{+}\right)
$$

which must satisfy the string equation

$$
[P, Q]=1
$$

Here $\tau=t_{0}$ is the coupling to the lowest dimension operator.

The defining property of $\psi_{ \pm}$is that they are the Baker-Akhiezer functions of the ZS integrable hierarchy. That is, they satisfy the differential equations

$$
Q\left(\begin{array}{c}
\psi_{+} \\
\psi_{-}
\end{array}\right)=x\left(\begin{array}{c}
\psi_{+} \\
\psi_{-}
\end{array}\right), \quad P\left(\begin{array}{c}
\psi_{+} \\
\psi_{-}
\end{array}\right)=-\partial_{x}\left(\begin{array}{c}
\psi_{+} \\
\psi_{-}
\end{array}\right)
$$

Comparing with (A.7), we see that if $Z_{ \pm}$have charge \pm 1 under the RR shift symmetry $\beta \rightarrow \beta+$ const (recall the discussion under (2.6)), then $\psi_{ \pm}$have definite charge $\pm 1 / 2$. This shows that these are indeed the partition functions of the charged FZZT branes. 0

For the special case of $(p, q)=(2,4)$ relevant to the paper, we set $t_{2}=-4, \tau=t_{0}=\mu$, and all other $t_{j}=0$. Then

$$
Q=\left(\begin{array}{ll}
i \partial_{\mu} & -\frac{i Z_{+}}{\sqrt{2}} \\
\frac{i Z_{-}}{\sqrt{2}} & -i \partial_{\mu}
\end{array}\right)
$$

6 We thank D. Gaiotto for help with the operator formalisms described in this subsection and the next.

7 When checking (A.10) against (A.9), one should keep in mind that since $P$ and $Q$ are differential operators in $\mu$, they commute with with $x$ and $\partial_{x}$. Thus $P Q \Psi=P(x \Psi)=x P \Psi=-x \partial_{x} \Psi$, and similarly for $Q P \Psi$. 
and the general formula for $P$ yields

$P=-4\left(Q\left(-Q^{2}\right)^{1 / 2}\right)_{+}+\mu\left(Q\left(-Q^{2}\right)^{-1 / 2}\right)_{+}=-i\left(\begin{array}{cc}4 \partial_{\mu}^{2}-r^{2}-\mu & -4 \frac{Z_{+}}{\sqrt{2}} \partial_{\mu}-\sqrt{2} Z_{+}^{\prime} \\ 4 \frac{Z_{-}}{\sqrt{2}} \partial_{\mu}+\sqrt{2} Z_{-}^{\prime} & -4 \partial_{\mu}^{2}+r^{2}+\mu\end{array}\right)$

One can check that $[P, Q]=1$ is equivalent to the string equation (2.2). It is also straightforward to check that the defining equations $($ A.10) are the same as the differential equations (4.2) studied in the paper.

\section{A.2. Type $0 A$}

The matrix model dual to $0 \mathrm{~A}$ minimal string theory is 13

$$
\tilde{\mathcal{Z}}_{N}(q)=\int d M d M^{\dagger} e^{-\operatorname{Tr} V\left(M M^{\dagger}\right)}
$$

where $M$ is a $(N+|q|) \times N$ complex matrix, and $V\left(M M^{\dagger}\right)$ is polynomial in $M M^{\dagger}$. By bringing $M$ to the form $M_{i j}=\sqrt{\lambda_{i}} \delta_{i j}$, we can reduce (A.1) down to an integration over eigenvalues:

$$
\tilde{\mathcal{Z}}_{N}(q)=\int_{0}^{\infty} d \lambda_{i} \lambda_{i}^{|q|} e^{-V\left(\lambda_{i}\right)} \Delta(\lambda)^{2}
$$

The model describing our system has $V(\lambda)=-\lambda+\tilde{g} \lambda^{2}$; the continuum limit consists of taking $N \rightarrow \infty$ while simultaneously tuning $\tilde{g}$ to its critical value. In this limit, $\tilde{\mathcal{Z}}_{N}$ becomes the bulk partition function $\mathcal{Z}(\mu, q)$ (the same as the $0 \mathrm{~B}$ partition function, in this special case), and the eigenvalues $\lambda_{i}$ become distributed on a single cut ending at the origin.

The partition function $\psi_{0}$ of the neutral FZZT brane is obtained from the doublescaling limit of the nearly orthonormal wavefunction

$$
\psi_{N}(\lambda, q)=\frac{1}{\sqrt{h_{N}}} e^{-V(\lambda) / 2}\left\langle\operatorname{det}\left(\lambda-M M^{\dagger}\right)\right\rangle
$$

By nearly orthonormal, we mean that the wavefunctions satisfy

$$
\int_{0}^{\infty} d \lambda \lambda^{|q|} \psi_{m}(\lambda, q) \psi_{n}(\lambda, q)=\delta_{m, n}
$$

In this limit

$$
\psi_{0}(x, q)=\lim _{N \rightarrow \infty} \psi_{N}\left(x^{2}, q\right)
$$


is the Baker-Akhiezer function of the integrable hierarchy underlying the $0 \mathrm{~A}$ minimal string. This integrable hierarchy belongs to the usual KP hierarchy of the bosonic string, but with a few important differences [8 12, 14]. Instead of Lax operators $P$ and $Q$, the operators here are

$$
Q=\partial_{\tau}^{2}-u
$$

( $\tau=t_{0}$ is again the coupling to the lowest-dimension operator, and $u=\partial_{\tau}^{2} \log \tilde{\mathcal{Z}}$ is the specific heat) and

$$
D=-\sum_{j \geq 0}\left(j+\frac{1}{2}\right) t_{2 j} Q_{+}^{j+1 / 2}
$$

with $Q$ and $D$ satisfying the string equation

$$
[D, Q]=Q
$$

The defining equations for $\psi_{0}$ are then

$$
Q \psi_{0}=-2 x^{2} \psi_{0}, \quad D \psi_{0}=\left(-\frac{1}{2} x \partial_{x}-\frac{1}{2}|q|-\frac{1}{4}\right) \psi_{0}
$$

Since $Q$ and $D$ are invariant under $\beta \rightarrow \beta+$ const, $\psi_{0}$ is indeed neutral under the $\mathrm{RR}$ symmetry.

For $(p, q)=(2,4)$, we set $\tau=t_{0}=\mu$ and $t_{2}=2 / 3$. Then the Lax operators are

$$
Q=\partial_{\mu}^{2}-u, \quad D=-\frac{1}{2} \mu \partial_{\mu}-\left(\partial_{\mu}^{3}-\frac{3}{4}\left\{u, \partial_{\mu}\right\}\right)
$$

One can check that with $u=r^{2}+\mu,[D, Q]=Q$ becomes equivalent to the string equation (2.3) after multiplying the equation by $r^{2}$ and integrating once with respect to $\mu$. By taking appropriate linear combinations of the two equations in (A.21), we can reduce them down to the form (5.2).

\section{A.3. More on the neutral brane in the complex matrix model}

Finally, let us analyze in more detail the matrix model description of the neutral brane, starting from (A.15). This is useful for a variety of reasons. First, we will see how the identity (5.4) can be easily derived using the matrix model. Second, we will confirm the symmetries and boundary conditions imposed at the end of section 5.1, as well as the asymptotics derived in section 5.2. For simplicity, we will assume that $q \in \mathbb{Z}$. 
To begin, let us note that $|q|$ can be increased to $|q|+1$ in the complex matrix model by inserting a determinant operator $\operatorname{det}\left(\lambda-M M^{\dagger}\right)$ at $\lambda=0$, since this has the effect of changing the measure from $\lambda^{|q|}$ to $\lambda^{|q|+1}$. Therefore, starting from the finite $N$ formula (A.15) in background $q$, we find that

$$
\psi_{N}(\lambda,|q|+1)=\frac{1}{\sqrt{h_{N}}} e^{-V(\lambda) / 2}\left\langle\operatorname{det}\left(-M M^{\dagger}\right)\right\rangle_{|q|}^{-1}\left\langle\operatorname{det}\left(\lambda-M M^{\dagger}\right) \operatorname{det}\left(-M M^{\dagger}\right)\right\rangle_{|q|}
$$

Here the expectation value is evaluated in background $|q|$. Using the determinant formulas in [40], this becomes

$$
\psi_{N}(\lambda,|q|+1) \sim \frac{1}{\lambda} \psi_{N}(0,|q|)^{-1}\left(\psi_{N+1}(\lambda,|q|) \psi_{N}(0,|q|)-\psi_{N}(\lambda,|q|) \psi_{N+1}(0,|q|)\right)
$$

The reason we can apply the determinant formulas of [40] is because they depend only on the orthogonality of the polynomials and not on the details of the measure or limits of integration.

In the double scaling limit, the shift in the index of the orthonormal wavefunction becomes a derivative with respect to $\mu$. Therefore, (A.24) becomes

$$
\psi_{N}\left(x^{2},|q|+1\right) \rightarrow \psi_{0}(x, \mu,|q|+1)=\frac{1}{x^{2}}\left(\partial_{\mu} \psi_{0}(x, \mu,|q|)-\psi_{0}(x, \mu,|q|) \partial_{\mu} \log \psi_{0}(0, \mu,|q|)\right)
$$

Finally, using the fact that $\psi_{0}(0, \mu,|q|)=Z_{+}(\mu,|q|)$ (see (5.9), and also the calculations below), we arrive at precisely the identity (5.4) in the text.

Although (5.4) is useful for relating $|q|$ to $|q|+1$, a more straightforward way to understand the $q$ dependence of the neutral brane is to relate everything directly to $q=0$. To do this, note that another way of writing (A.15) is:

$$
\psi_{N}(\lambda, q)=\frac{1}{\sqrt{h_{N}}} e^{-V(\lambda) / 2}\left\langle\left(\operatorname{det}\left(-M M^{\dagger}\right)\right)^{|q|}\right\rangle^{-1}\left\langle\operatorname{det}\left(\lambda-M M^{\dagger}\right)\left(\operatorname{det}\left(-M M^{\dagger}\right)\right)^{|q|}\right\rangle
$$

where now \langle\rangle denotes the expectation value taken in a background of $q=0$. Using the determinant formulas in [40], this becomes

$$
\psi_{N}(\lambda, q)=\left(\frac{\operatorname{det}_{k, l} \psi_{N+k-1}\left(\lambda_{l}, 0\right)}{\operatorname{det}_{k, l} \lambda_{l}^{k-1}}\right)^{-1}\left(\frac{\operatorname{det}_{i, j} \psi_{N+i-1}\left(\lambda_{j}, 0\right)}{\operatorname{det}_{i, j} \lambda_{j}^{i-1}}\right)
$$

where $1 \leq i, j \leq|q|+1 ; 1 \leq k, l \leq|q| ; \lambda_{1}=\ldots=\lambda_{|q|}=0$; and $\lambda_{|q|+1}=\lambda$. Therefore, in the double-scaling limit (A.27) becomes

$$
\psi_{N}\left(x^{2}, q\right) \rightarrow \psi_{0}(x, \mu, q)=\left(\frac{\operatorname{det}_{k, l} \partial_{\mu}^{l-1} \psi_{0}\left(\sqrt{\lambda_{k}}, \mu, q=0\right)}{\operatorname{det}_{k, l} \lambda_{k}^{l-1}}\right)^{-1}\left(\frac{\operatorname{det}_{i, j} \partial_{\mu}^{j-1} \psi_{0}\left(\sqrt{\lambda_{i}}, \mu, q=0\right)}{\operatorname{det}_{i, j} \lambda_{i}^{j-1}}\right)
$$


Here $i, j, k, l$ are as in (A.27); $\lambda_{1}=\ldots=\lambda_{|q|}=0$; and $\lambda_{|q|+1}=x^{2}$. The limit to $\lambda_{1}=\ldots=\lambda_{|q|}=0$ is slightly subtle - it involves the fact (see (5.9)) that $\psi_{0}$ depends only on $x^{2}$ around $x=0$.

Eq. (A.28) is a completely general formula for the partition function of the neutral brane, valid for all $(2,2 k) 0 \mathrm{~A}$ multicritical points. Since the only input is the neutral brane partition function at $q=0$, we can use (A.28) to learn about the system at nonzero $q$, at least in principle. This can be difficult in practice, however, since the formula in its full generality is rather complicated. Fortunately, it simplifies and becomes more useful in the various limits studied in section 5.1.

For instance, a straightforward calculation shows that in the semiclassical limit, (A.28) reduces to

$$
\psi_{0}(x, \mu, q) \approx(z(x, \mu)-z(0, \mu))^{|q|} x^{-2|q|} \psi_{0}(x, \mu, q=0)
$$

where

$$
z(x, \mu) \equiv \partial_{\mu} D(x, \mu)
$$

and $D(x, \mu)$ is the WKB exponent of $\psi_{0}$ at $q=0$. To derive (A.29), use the fact that $\psi_{0} \approx e^{D(x, \mu)+\ldots}$ in the semiclassical limit, implying that

$$
\partial_{\mu}^{m} \psi_{0} \approx z^{m} \psi_{0}
$$

to leading order. Again, let us emphasize that (A.29) is completely general, valid for all $(2,2 k) 0 \mathrm{~A}$ models. The only input is $D(x, \mu)$, which can be derived from the boundary state of the $q=0, \eta=+1$ FZZT brane. For the case of $(2,4)$ considered in this paper, our results in section 3 imply

$$
z(x, \mu)= \begin{cases}\sqrt{2} i x & \mu<0 \\ -\sqrt{2} \sqrt{\frac{\mu}{2}-x^{2}} & \mu>0\end{cases}
$$

Substituting this into (A.29), we reproduce precisely the $q$-dependence of the asymptotics (5.7) and (5.8).

It is also just as straightforward to compare the general matrix model formula (A.28) with the small $x$ limit (5.9). In this limit, we find the simple result from the matrix model:

$$
\psi_{0}(x, \mu, q) \rightarrow \frac{\tilde{\mathcal{Z}}(\mu,|q|+1)}{\tilde{\mathcal{Z}}(\mu,|q|)}
$$

This formula is general, valid for all $(2,2 k)$. For the special case of $(2,4)$, we have $\tilde{\mathcal{Z}}(\mu, q)=$ $\mathcal{Z}(\mu, q)$. And for $0 \mathrm{~B}$ we have the identity (2.10), $\mathcal{Z}(\mu, q+1)=\mathcal{Z}(\mu, q) Z_{+}(\mu, q)$. When substituted into (A.33), this gives precisely the small $x$ asymptotics (5.9) which are smooth at $x=0$. 


\section{Appendix B. Proving some useful identities}

\section{B.1. Closed-string sector}

In this appendix, we will analyze more carefully various identities used in the text. Let us start with the closed-string sector and explore in more detail the properties of the string equation (2.2). In particular, we wish to justify the treatment of the integration constants alluded to in footnote 2. The issue is that since the string equations (2.2) depend only on $\beta^{\prime}$, the function $\beta$ is determined up to an additive $q$ dependent integration constant. This ambiguity amounts to freedom to rescale $Z_{ \pm}(\mu, q)$ by $B(q)^{ \pm 1}$ with $B$ an (almost) arbitrary function of $q . B(q)$ is obviously constrained by the charge conjugation symmetry $Z_{+}(\mu, q)=Z_{-}(\mu,-q)$, which implies that $B(q)=B(-q)^{-1}$, and $B(0)=1$. The question is what other constraints we must impose on $B(q)$ in order to determine it completely.

The solutions of the equations for different values of $q$ are related by 8

$$
r(\mu, q \pm 1)^{2}=r(\mu, q)^{2}-2 \partial_{\mu}^{2} \log Z_{ \pm}(\mu, q)
$$

or equivalently by

$$
r(\mu, q \pm 1)^{2}=-r(\mu, q)^{2}-2 \mu+2\left(\partial_{\mu} \log Z_{ \pm}(\mu, q)\right)^{2}
$$

The proof of the first identity was sketched in the text, and the second identity is trivially related to the first through the definition of $Z_{ \pm}$(2.7) and the string equation (2.3). Using the symmetry $r(\mu, q)=r(\mu,-q)$ in (B.1), we find

$$
\partial_{\mu}^{2} \log \left(Z_{+}(\mu, q-1) Z_{-}(\mu, q)\right)=0
$$

Integrating twice, we find

$$
Z_{+}(\mu, q-1) Z_{-}(\mu, q)=a(q) e^{b(q) \mu}
$$

where $a(q)$ and $b(q)$ are integration constants. It is evident from the asymptotic expansions in section 2.2 that $b(q)=0$. Then, using the freedom to rescale $Z_{ \pm}$by $B(q)^{ \pm 1}$ we can set $a(q)=1$. We conclude that

$$
Z_{+}(\mu, q-1) Z_{-}(\mu, q)=1
$$

8 Note that these identities might not be consistent with the physically acceptable boundary conditions on $r(\mu, q)$ at $\mu \rightarrow \pm \infty$. This is discussed more in the text. 
as claimed in the text. Using (2.1) and the same integration constants as in (B.5), we can also integrate (B.1) twice to conclude

$$
Z_{ \pm}(\mu, q)=e^{F(\mu, q)-F(\mu, q \pm 1)}=\frac{\mathcal{Z}(\mu, q \pm 1)}{\mathcal{Z}(\mu, q)}
$$

which reproduces $(2.10)$ in the text.

The identities presented here can be written a number of different ways, some of which may be more familiar to others. For instance, if we define

$$
f_{ \pm} \equiv \partial_{\mu} \log Z_{ \pm}=\frac{r^{\prime}}{r} \mp \beta^{\prime}=\frac{r^{\prime}}{r} \mp \frac{q}{r^{2}}
$$

then, using (2.2) or (2.3) we readily find that they satisfy another version of the Painleve II equation

$$
f_{ \pm}^{\prime \prime}+2 \mu f_{ \pm}-2 f_{ \pm}^{3}= \pm 2 q+1
$$

These solutions are related by

$$
f_{+}^{2}+f_{+}^{\prime}=f_{-}^{2}+f_{-}^{\prime}
$$

Another way of writing (2.3) is in terms of the specific heat $v=F^{\prime \prime}=\frac{1}{2} r^{2}$, which satisfies

$$
v^{\prime \prime}-\frac{\left(v^{\prime}\right)^{2}}{2 v}-4 v^{2}-2 \mu v+\frac{q^{2}}{2 v}=0
$$

This is known as the Painlevé 34 equation, and $f_{ \pm}=\frac{v^{\prime} \mp q}{2 v}$. Finally, in terms of $f_{ \pm}$the relation (B.1) between the values with different $q$ is known as the Miura transformation,

$$
f_{ \pm}(\mu, q)^{2}-\partial_{\mu} f_{ \pm}(\mu, q)=f_{ \pm}(\mu, q \pm 1)^{2}+\partial_{\mu} f_{ \pm}(\mu, q \pm 1)
$$

which is a consequence of (B.8).

\section{B.2. Open-string sector}

Next let us turn to the open-string sector. We wish to sketch a proof of (4.5) in the text:

$$
\psi_{-}(x, \mu, q)=Z_{-}(\mu, q) \psi_{+}(x, \mu, q-1)
$$

Our strategy for the proof will be to explicitly construct a solution of the differential equations (4.2) that obeys (B.12). 
The first step of the proof is define a function $S=S(x, \mu, q)$ via the differential equations

$$
\begin{aligned}
0=\partial_{\mu} \log S- & \left(2 i x-\partial_{\mu} \log Z_{-}\right)-\frac{1}{\sqrt{2}} S^{-1}+\frac{1}{\sqrt{2}} r^{2} S \\
0=\partial_{x} \log S- & 2 i\left(4 x^{2}+r^{2}+\mu\right)+i\left(2 \sqrt{2} i x+\sqrt{2} \partial_{\mu} \log Z_{-}\right) S^{-1} \\
- & i\left(2 \sqrt{2} i x-\sqrt{2} \partial_{\mu} \log Z_{+}\right) r^{2} S
\end{aligned}
$$

Using the identities in section 2.1, one can show that these differential equations are compatible with the recursion relation

$$
r(\mu, q-1)^{2} S(x, \mu, q-1)=\left(2 \sqrt{2} i x-\sqrt{2} \partial_{\mu} \log Z_{-}(\mu, q)\right)+S(x, \mu, q)^{-1}
$$

In other words, one can show that starting from a solution $S\left(x, \mu, q_{0}\right)$ of (B.13) at some $q=q_{0}$, the functions $S(x, \mu, q)$ obtained through (B.14) also satisfy (B.13) at $q$.

The next step is to introduce the function $h=h(x, \mu, q)$ which satisfies

$$
\begin{aligned}
& \partial_{\mu} \log h=-i x+\frac{1}{\sqrt{2}} r^{2} S \\
& \partial_{x} \log h=-i\left(4 x^{2}+\mu\right)+\left(2 \sqrt{2} x Z_{+}+i \sqrt{2} Z_{+}^{\prime}\right) Z_{-} S-i r^{2}
\end{aligned}
$$

One can check that

$$
S(x, \mu, q)=\frac{h(x, \mu, q-1)}{h(x, \mu, q)}
$$

and that

$$
\Psi=h(x, \mu, q)\left(\begin{array}{c}
1 \\
Z_{-}(\mu, q) S(x, \mu, q)
\end{array}\right)
$$

satisfies $Q \Psi=x \Psi$ and $P \Psi=-\partial_{x} \Psi$ for all $q$. This completes the proof of (B.12). 


\section{References}

[1] D. J. Gross and E. Witten, "Possible Third Order Phase Transition In The Large N Lattice Gauge Theory," Phys. Rev. D 21, 446 (1980).

[2] V. Periwal and D. Shevitz, "Unitary Matrix Models As Exactly Solvable String Theories," Phys. Rev. Lett. 64, 1326 (1990); V. Periwal and D. Shevitz, "Exactly Solvable Unitary Matrix Models: Multicritical Potentials And Correlations," Nucl. Phys. B 344, 731 (1990).

[3] C. R. Nappi, "Painleve-II And Odd Polynomials," Mod. Phys. Lett. A 5, 2773 (1990).

[4] C. Crnkovic, M. R. Douglas and G. W. Moore, "Physical Solutions For Unitary Matrix Models," Nucl. Phys. B 360, 507 (1991).

[5] C. Crnkovic, M. R. Douglas and G. W. Moore, "Loop equations and the topological phase of multi-cut matrix models," Int. J. Mod. Phys. A 7, 7693 (1992) arXiv:hep th/9108014].

[6] T. J. Hollowood, L. Miramontes, A. Pasquinucci and C. Nappi, "Hermitian versus anti-Hermitian one matrix models and their hierarchies," Nucl. Phys. B 373, 247 (1992) arXiv:hep-th/9109046.

[7] R. C. Brower, N. Deo, S. Jain and C. I. Tan, "Symmetry breaking in the double well Hermitian matrix models," Nucl. Phys. B 405, 166 (1993) arXiv:hep-th/9212127.

[8] T. R. Morris, "2-D Quantum Gravity, Multicritical Matter And Complex Matrices," FERMILAB-PUB-90-136-T

[9] S. Dalley, C. V. Johnson and T. Morris, "Multicritical complex matrix models and nonperturbative 2-D quantum gravity," Nucl. Phys. B 368, 625 (1992).

[10] S. Dalley, C. V. Johnson, T. R. Morris and A. Watterstam, "Unitary matrix models and 2-D quantum gravity," Mod. Phys. Lett. A 7, 2753 (1992) arXiv:hep-th/9206060.

[11] R. Lafrance and R. C. Myers, "Flows For Rectangular Matrix Models," Mod. Phys. Lett. A 9, 101 (1994) arXiv:hep-th/9308113.

[12] P. Di Francesco, "Rectangular Matrix Models and Combinatorics of Colored Graphs," Nucl. Phys. B 648, 461 (2003) arXiv:cond-mat/0208037.

[13] I. R. Klebanov, J. Maldacena and N. Seiberg, "Unitary and complex matrix models as 1-d type 0 strings," Commun. Math. Phys. 252, 275 (2004) arXiv:hep-th/0309168].

[14] C. V. Johnson, "Non-perturbative string equations for type 0A," arXiv:hep-th/0311129.

[15] N. Seiberg and D. Shih, "Branes, rings and matrix models in minimal (super)string theory," JHEP 0402, 021 (2004) arXiv:hep-th/0312170.

[16] C. V. Johnson, "Tachyon condensation, open-closed duality, resolvents, and minimal bosonic and type 0 strings," arXiv:hep-th/0408049.

[17] D. Gaiotto, L. Rastelli and T. Takayanagi, "Minimal superstrings and loop gas models," arXiv:hep-th/0410121. 
[18] A. Kapustin, "A remark on worldsheet fermions and double-scaled matrix models," arXiv:hep-th/0410268.

[19] H. Kawai, T. Kuroki and Y. Matsuo, "Universality of nonperturbative effect in type 0 string theory," arXiv:hep-th/0412004.

[20] M. R. Douglas, I. R. Klebanov, D. Kutasov, J. Maldacena, E. Martinec and N. Seiberg, "A new hat for the $c=1$ matrix model," arXiv:hep-th/0307195.

[21] D. Kutasov, K. Okuyama, J. w. Park, N. Seiberg and D. Shih, "Annulus amplitudes and ZZ branes in minimal string theory," JHEP 0408, 026 (2004) arXiv:hepth/0406030|.

[22] A. B. Zamolodchikov and A. B. Zamolodchikov, "Liouville field theory on a pseudosphere," arXiv:hep-th/0101152.

[23] T. Fukuda and K. Hosomichi, "Super Liouville theory with boundary," Nucl. Phys. B 635, 215 (2002) arXiv:hep-th/0202032.

[24] C. Ahn, C. Rim and M. Stanishkov, "Exact one-point function of $\mathrm{N}=1$ super-Liouville theory with boundary," Nucl. Phys. B 636, 497 (2002) arXiv:hep-th/0202043].

[25] V. Fateev, A. B. Zamolodchikov and A. B. Zamolodchikov, "Boundary Liouville field theory. I: Boundary state and boundary two-point function," arXiv:hep-th/0001012.

[26] need to supply reference TeschnerMD.

[27] A. Sen, "Tachyon dynamics in open string theory," arXiv:hep-th/0410103.

[28] J. Maldacena, G. W. Moore, N. Seiberg and D. Shih, "Exact vs. semiclassical target space of the minimal string," arXiv:hep-th/0408039.

[29] V. G. Kac and M. Wakimoto, "Exceptional hierarchies of soliton equations," Proc. Sympos. Pure Math. 49, 191 (1989).

[30] P. Horava, "Type IIA D-branes, K-theory, and matrix theory," Adv. Theor. Math. Phys. 2, 1373 (1999) arXiv:hep-th/9812135.

[31] A. R. Its and V. Y. Novokshenov, "The Isomonodromic Deformation Method in the Theory of Painlevé Equations," Springer-Verlag (1986).

[32] P. Bleher and A. Its, "Double scaling limit in the random matrix model: the RiemannHilbert approach," arXiv:math-ph/0201003.

[33] A. R. Its and V. Yu. Novokshenov, "Effective sufficient conditions for the solvability of the inverse problem of monodromy theory for systems of linear ordinary differential equations," Funct. Anal. Appl. 22, 190 (1988)

[34] M. Jimbo, T. Miwa and K. Ueno, "Monodromy preserving deformation of linear orderinary differential equations with rational coefficients, I," Physica D 2, 306 (1981)

[35] J. M. Maldacena, G. W. Moore and N. Seiberg, "D-brane instantons and K-theory charges," JHEP 0111, 062 (2001) arXiv:hep-th/0108100.

[36] G. W. Moore, "Geometry Of The String Equations," Commun. Math. Phys. 133, 261 (1990). 
[37] G. W. Moore, "Matrix Models Of 2-D Gravity And Isomonodromic Deformation," Prog. Theor. Phys. Suppl. 102, 255 (1990).

[38] H. Flaschka and A. C. Newell, "Monodromy And Spectrum Preserving Deformations. 1," Commun. Math. Phys. 76, 65 (1980).

[39] A. R. Its, A. A. Kapaev, "The Irreducibility of the Second Painleve Equation and the Isomonodromy Method," in Toward the exact WKB analysis of differential equations, linear or non-linear, C. Howls, T. Kawai and Y. Takei, eds., Kyoto University Press (2000).

[40] A. Morozov, "Integrability And Matrix Models," Phys. Usp. 37, 1 (1994) arXiv:hepth/9303139]. 